University of Wollongong

Research Online

Faculty of Engineering and Information

Faculty of Engineering and Information

Sciences - Papers: Part A

Sciences

$1-1-2015$

Privacy-preserving ciphertext multi-sharing control for big data storage

Kaitai Liang

Aalto University, kaitai.liang@aalto.fi

Willy Susilo

University of Wollongong,wsusilo@uow.edu.au

Joseph K. Liu

Monash University, ksliu@i2r.a-star.edu.sg

Follow this and additional works at: https://ro.uow.edu.au/eispapers

Part of the Engineering Commons, and the Science and Technology Studies Commons

Research Online is the open access institutional repository for the University of Wollongong. For further information contact the UOW Library: research-pubs@uow.edu.au 


\title{
Privacy-preserving ciphertext multi-sharing control for big data storage
}

\author{
Abstract \\ The need of secure big data storage service is more desirable than ever to date. The basic requirement of \\ the service is to guarantee the confidentiality of the data. However, the anonymity of the service clients, \\ one of the most essential aspects of privacy, should be considered simultaneously. Moreover, the service \\ also should provide practical and fine-grained encrypted data sharing such that a data owner is allowed to \\ share a ciphertext of data among others under some specified conditions. This paper, for the first time, \\ proposes a privacy-preserving ciphertext multi-sharing mechanism to achieve the above properties. It \\ combines the merits of proxy re-encryption with anonymous technique in which a ciphertext can be \\ securely and conditionally shared multiple times without leaking both the knowledge of underlying \\ message and the identity information of ciphertext senders/recipients. Furthermore, this paper shows \\ that the new primitive is secure against chosen-ciphertext attacks in the standard model.

\section{Disciplines} \\ Engineering | Science and Technology Studies \\ Publication Details \\ Liang, K., Susilo, W. \& Liu, J. K. (2015). Privacy-preserving ciphertext sharing mechanism for big data \\ storage. IEEE Transactions on Information Forensics and Security, 10 (8), 1578-1589.
}




\title{
Privacy-Preserving Ciphertext Multi-Sharing Control for Big Data Storage
}

\author{
Kaitai Liang, Willy Susilo, Senior Member, IEEE and Joseph K. Liu ${ }^{+}$
}

\begin{abstract}
The need of secure big data storage service is more desirable than ever to date. The basic requirement of the service is to guarantee the confidentiality of the data. However, the anonymity of the service clients, one of the most essential aspects of privacy, should be considered at the same time. Moreover, the service also should provide practical and finegrained encrypted data sharing so that a data owner is allowed to share a ciphertext of data among others based on his/her wish, under some pre-defined conditions. This paper, for the first time, proposes a privacy-preserving ciphertext multi-sharing mechanism to achieve the above properties simultaneously. It combines the merits of proxy re-encryption with anonymous technique in which a ciphertext can be securely and conditionally shared multiple times without leaking both the knowledge of underlying plaintext and the identity information of ciphertext senders/recipients. Furthermore, the paper shows that the new primitive is secure against chosen-ciphertext attacks in the standard model under the Decisional $\mathcal{P}$ Bilinear Diffie-Hellman assumption.
\end{abstract}

Keywords: Privacy, anonymity, proxy re-encryption, big data.

\section{INTRODUCTION}

To date many individuals and companies choose to upload their data to clouds since the clouds are able to support considerable data storage service but also efficient data processing capability. Accordingly, it is unavoidable that trillion tons of personal and industrial data are flooded in the Internet. For example, in some smart grid scenario a governmental department might choose to supervise the electricity consumption of a local living district. A great amount of electricity consumed date of each family located inside the district will be automatically transferred to the department via Internet period by period. The need of big data storage, therefore, is more desirable than ever.

A basic security requirement of big data storage is to guarantee the confidentiality of the data. Fortunately, some existing cryptographic encryption mechanisms can be employed to fulfill the requirement. For instance, Public Key Encryption (PKE) allows a data sender to encrypts the data under the public key of receiver such that no one except the valid

Joseph K. Liu is the corresponding author.

K. Liang is with the Department of Information and Computer Science, School of Science and Technology, Aalto University, Finland (e-mail kaitai.liang@aalto.fi).

W. Susilo, is with Centre for Computer and Information Security Research, School of Computer Science and Software Engineering, University of Wollongong, Wollongong, NSW 2522, Australia (e-mail: wsusilo@uow.edu.au).

J.K. Liu is with Institute for Infocomm Research, A*STAR, Singapore (email: ksliu@i2r.a-star.edu.sg). recipient can gain access to the data. Nevertheless, this does not satisfy all the needs behind the scenario of big data storage.

Consider the following scenario. We suppose a hospital stores its patients' medical records in a cloud storage system and meanwhile, the records are all in the encrypted form so as to avoid the cloud server from accessing to any plain medical information. After a medical record is encrypted and further uploaded to the cloud, only those specified doctors can gain access to the record. By using some traditional PKE, Identity-Based Encryption (IBE), or Attribute-Based Encryption (ABE), the confidentiality of the record can be protected effectively.

By trivially employing traditional encryption mechanisms, nevertheless, we cannot prevent some sensitive medical information from being leaked to the cloud server but also the public. This is so because traditional encryption systems do not consider the anonymity of a ciphertext sender/receiver. Accordingly, someone, could be anyone with capability to access the ciphertext of the record, may know whose public key the ciphertext is encrypted under, namely who is the owner of the ciphertext, so that the patient associated with the ciphertext can be easily identified. Similarly, the recipient/destination of the ciphertext, e.g., Cardiology Dept., can be known from the ciphertext without any difficulty as well. This seriously disgraces the privacy of the patient.

Moreover, a patient might be transferred to different medical departments in his/her treatment phase. The corresponding medical record then needs to be converted to the ciphertexts corresponding to various receivers so as to be shared among the departments. Therefore, the update of ciphertext recipient is desirable. Precisely speaking, a fine-grained ciphertext update for receivers is necessary in the sense that a ciphertext could be conditionally shared with others. The medical record owner, i.e. the patient, has rights to decide who can gain access to his/her record, and which kinds of data could be accessed. For example, the patient can choose to specify that only the medical record described with "teeth" can be read by a dentist. This fine-grained control avoids a data sharing mechanism from being limited to the "all-or-nothing" share mode.

This work is trying to solve the above problems. To preserve anonymity, some well-known encryption mechanisms are proposed in the literature, such as anonymous IBE [8], anonymous ABE [40]. By employing these primitives, the source and the destination of data can be protected privately. But the primitives cannot support the update of ciphertext receiver.

There are some naive approaches to update ciphertext's recipient. For instance, data owner can employ the decrypt-thenre-encrypt mode. However, this is applicable to the scenario 
where there is only a small amount of data. If the encrypted data is either a group of sequences of gene information or a network audit log, the decryption and re-encryption might be time consumed and computation costly. Moreover, this mode also suffers from a limitation that the data owner has to be online all the time. Alternatively, a fully trusted third party with knowledge of the decryption key of the data owner may be delegated to handle the task. Nevertheless, this strongly relies on the trust of the party. Besides, the anonymity of the receiver cannot be achieved as the party needs to know the information of recipient to proceed the re-encryption. Therefore, both of the approaches do not scale well.

Introduced by Mambo and Okamoto [27] and first defined in [5], Proxy Re-Encryption (PRE) is proposed to tackle the dilemma of data sharing. It allows a semi-trusted party called proxy to transform a ciphertext intended for a user into a ciphertext of the same plaintext intended for another user without leaking knowledge of either the decryption keys or the plaintext. The workload of data owner is transferred to the proxy, and the "on-line all the time" requirement for the owner is unnecessary.

This work focuses on the identity-based cryptographic setting. To employ PRE in the IBE setting, [17] defined the notion of Identity-Based Proxy Re-Encryption (IBPRE), which offers a practical solution for access control in networked file storage [17], and secure email with IBE [17]. To capture privacy-preserving property and ciphertext's recipient update simultaneously, [31] proposed an anonymous IBPRE system, which is CCA security in the random oracle model.

This valuable research work introduces the first anonymous IBPRE in the literature and meanwhile, it leaves us interesting and meaningful open problems. The work only supports one-time ciphertext receiver update, while multiple receivers update is desirable in practice. On the other hand, the work provides an "all-or-nothing" share mode that limits the flexibility of data sharing.

\section{A. Our Contributions}

In this paper, we are trying to propose a ciphertext sharing mechanism with the following properties:

- Anonymity: given a ciphertext, no one knows the corresponding sender and receiver.

- Multiple receiver-update: given a ciphertext, the receiver of the ciphertext can be updated in multiple times. In this paper, we refer to this property as "multi-hop".

- Conditional sharing: a ciphertext can be fine-grained shared with others if the pre-specified conditions are satisfied.

Achievements. We investigate a new notion, AMHIBCPRE. We formalize the definition and security model by incorporating the definitions in [32], [33]. In the security model, we allow the corrupted users to be adaptively chosen by an adversary, while the adversary must output the challenge identity at the outset of security game. Moreover, we define four security models for different purposes. They are briefly described as follows:
- The security model of MH-IBCPRE is the basic model, in which a challenger plays the game with the adversary to launch CCA to the original ciphertext and re-encrypted ciphertext in order to solve a hard problem.

- We also consider the case where a proxy colludes with delegatee to compromise the underlying plaintext and the secret key of delegator. Here, the protection of the plaintext is difficult to achieve as the delegatee can decrypt the corresponding ciphertext. The secret key of the delegator, however, is possible to be secured.

- For the definition of collusion attacks model, we allow the adversary to acquire all re-encryption keys and the adversary wins the game if it outputs a valid secret key of an uncorrupted user. We note that our definition is in a selective model in which the adversary has to output a target identity at the outset of the security game.

- As to the security model of anonymity, it is more complicated in the sense that we categorize the game into two sub-games: one is the anonymity for the delegator (i.e. given the original ciphertext the adversary cannot output identity of the delegator), the other is the anonymity of the re-encryption key (i.e. the adversary cannot distinguish a valid re-encryption key from a random one belonging to the re-encryption key space).

We next propose a concrete construction for unidirectional AMH-IBCPRE, in which it achieves multiple ciphertext receiver update, conditional data sharing, anonymity and collusion-safe (i.e. holding against collusion attacks) simultaneously in the asymmetric bilinear map setting. Note the functionality of our system is generally described in Fig 1 . We state that our new primitive is applicable to many realworld applications, such as secure email forwarding, electronic encrypted data sharing, where both anonymity and flexible encrypted conversion are needed. We also show our scheme is CCA-secure in the standard model under the decisional $P$-Bilinear Diffie-Hellman assumption. To the best of our knowledge, our AMH-IBCPRE is the first of its kind in the literature.

\section{B. Related Work}

Following the concept of delegation of decryption rights introduced by Mambo and Okamoto [27], Blaze et al. [5] formalized the concept of proxy re-encryption and proposed a seminal bidirectional PRE scheme. Afterwards, many PRE schemes have been proposed, such as [2], [3], [11], [18], [25], [20], [23], [26], [21].

Employing traditional PRE in the IBE setting, Green and Ateniese [17] first defined the notion of IBPRE and proposed two unidirectional IBPRE schemes in the random oracle model: one is CPA secure and the other holds against CCA. Later, two CPA-secure IBE-PRE schemes (in the types of PKE-IBE and IBE-IBE) [28] have been proposed. In 2008, Tang et al. [35] proposed an IBPRE scheme with CPA security in the random oracle model, in which the delegator and the delegatee can come from different domains. Afterwards, Wang et al. proposed two IBPRE schemes that are both collusion-safe and non-transferable in the random oracle model. However, 


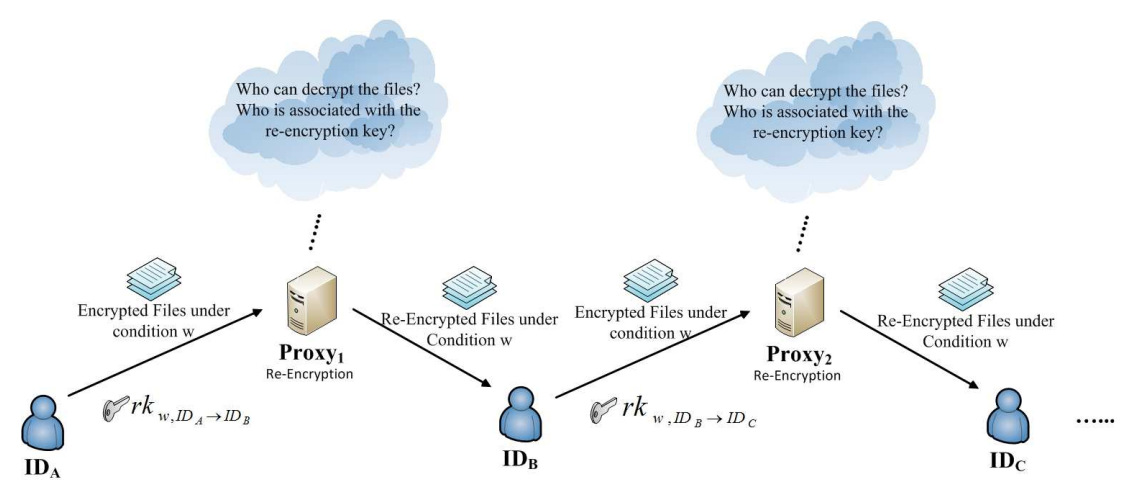

Fig. 1: Anonymous Multi-Hop Identity-Based Conditional Proxy Re-Encryption

the generation of re-encryption key requires the participation of the PKG. With the same technique, a CPA-secure IBPRE scheme (in the type of IBE-PKE) without random oracles was proposed by Minzuno and Doi [29]. Recently, an IBPRE with revocability and hierarchical confidentialities was proposed by Wang et al. [37]. Despite the scheme combines the IBPRE with proxy decryption, it still requires an interaction for reencryption key generation.

In the multiple ciphertext receiver update ${ }^{1}$ scenario, Green and Ateniese [17] proposed the first MH-IBPRE scheme which is CPA secure in 2007. Later, a RCCA-secure MH-IBPRE scheme without random oracles was proposed by $\mathrm{Chu}$ and Tzeng [12]. In 2010, Wang et al. [36] proposed the first CCAsecure MH-IBPRE with random oracles. These three schemes, however, are not collusion-safe. To solve the problem, Shao and Cao [32] proposed the first CCA-secure MH-IBPRE in the standard model with collusion-safe property.

To preserve the anonymity, the following cryptosystems are proposed in the literature. To hide the information revealed by the re-encryption key, Ateniese et al. [1] first define the notion of key-privacy (i.e. an adversary cannot identify delegator and delegatee even given re-encryption key) and proposed a CPA-secure scheme in the standard model. Later, Shao et al. [34] revised the security model of key privacy defined in [1] and proposed a single-hop unidirectional PRE scheme with CCA security in the standard model. To prevent from being traceable, Emura et al. [15] proposed a unidirectional IBPRE scheme for multi-hop setting, in which an adversary cannot identify the source from the destination ciphertext. The scheme is proven to be CCA secure in the random oracle model. To protect the privacy of both delegator and delegatee, Shao et al. [33] proposed the first Anonymous PRE (ANO-PRE) scheme which is CCA secure with collusionsafe in the random oracle model. The scheme ensures that an adversary cannot identify the recipient of original and reencrypted ciphertext even given the re-encryption key. In 2012, Shao [31] also proposed the first anonymous IBPRE with CCA security in the random oracle model.

In the identity-based and attribute-based encryption settings, some well-known systems supporting anonymity that have been proposed, such as [8], [9], [19], [9], [16], [40], [30].

\footnotetext{
${ }^{1}$ Note we refer to multiple ciphertext receiver update to a property called Multi-Hop (MH) in this paper.
}

However, we will focus on the combination of anonymity and ciphertext update properties. Therefore, the aforementioned systems will not be taken in comparison below.

Below we compare our work with the some related research works, and summarize the comparison of properties in Table I. While multiple ciphertext receiver update (denoting as M.U.), conditional share, collusion resistance (denoting as C.R.), anonymity, and without random oracle (denoting as W.R.O.), have all five been partially achieved by previous schemes, there is no efficient CCA-secure proposal that achieves all properties simultaneously in the standard model. This paper, for the first time, fills the gap.

TABLE I: Property Comparison

\begin{tabular}{|r|c|c|c|c|c|c|}
\hline Sch. & Security & W.R.O. & M.U. & C.R. & $\begin{array}{c}\text { Conditional } \\
\text { Share }\end{array}$ & Anonymity \\
\hline$[17]$ & CPA & X & $\checkmark$ & X & X & X \\
\hline$[12]$ & RCCA & $\checkmark$ & $\checkmark$ & X & X & Х \\
\hline$[32]$ & CCA & $\checkmark$ & $\checkmark$ & $\checkmark$ & X & X \\
\hline$[31]$ & CCA & X & X & $\checkmark$ & X & $\checkmark$ \\
\hline Ours & CCA & $\checkmark$ & $\checkmark$ & $\checkmark$ & $\checkmark$ & $\checkmark$ \\
\hline
\end{tabular}

\section{Definition And Security Models}

\section{A. Definition for Multi-Hop Identity-Based Conditional Proxy Re-Encryption}

Definition 1: A unidirectional Multi-Hop Identity-Based Conditional Proxy Re-Encryption (MH-IBCPRE) scheme consists of the following algorithms:

1) $(m p k, m s k) \leftarrow \operatorname{Setup}\left(1^{k}\right)$ : on input a security parameter $k$, output a master public key $m p k$ and a master secret key $m s k$. For simplicity, we omit $m p k$ in the expression of the following algorithms.

2) $s k_{I D} \leftarrow \operatorname{KeyGen}(m s k, I D)$ : on input $m s k$, and an identity $I D \in\{0,1\}^{*}$, output a secret key $s k_{I D}$.

3) $r k_{w, I D_{i} \rightarrow I D_{i^{\prime}}} \leftarrow \operatorname{ReKeyGen}\left(I D_{i}, s k_{I D_{i}}, I D_{i^{\prime}}, w\right)$ : on input a delegator's identity $I D_{i}$ and the corresponding secret key $s k_{I D_{i}}$, a delegatee's identity $I D_{i^{\prime}}$, and a condition $w \in\{0,1\}^{*}$, output a re-encryption key $r k_{w, I D_{i} \rightarrow I D_{i^{\prime}}}$ from $I D_{i}$ to $I D_{i^{\prime}}$ under condition $w$.

4) $C_{1, I D_{i}, w} \leftarrow \operatorname{Enc}\left(I D_{i}, w, m\right)$ : on input an identity $I D_{i}$, a condition $w$ and a message $m$, output a 1-level ciphertext $C_{1, I D_{i}, w}$ under identity $I D_{i}$. 
5) $C_{l+1, I D_{i^{\prime}}, w} \leftarrow \operatorname{Re} \operatorname{Enc}\left(r k_{w, I D_{i} \rightarrow I D_{i^{\prime}}}, w, C_{l, I D_{i}, w}\right)$ : on input $r k_{w, I D_{i} \rightarrow I D_{i^{\prime}}}$, a condition $w$ and an $l$-level ciphertext $C_{l, I D_{i}, w}$ under identity $I D_{i}$ and $w$, output an $(l+1)$ level ciphertext $C_{l+1, I D_{i^{\prime}}, w}$ under identity $I D_{i^{\prime}}$ and $w$ or $\perp$ for failure, where $l \geq 1, l \in \mathbb{N}$.

6) $m \leftarrow \operatorname{Dec}\left(s k_{I D_{i}}, w, C_{l, I D_{i}, w}\right)$ : on input $s k_{I D_{i}}, w$ and an $l$-level ciphertext $C_{l, I D_{i}, w}$ under identity $I D_{i}$ and $w$, output a message $m$ or $\perp$ for failure, where $l \geq 1, l \in \mathbb{N}$.

Correctness: For any $k, l \in \mathbb{N}$, any identities $I D_{i}, I D_{i^{\prime}} \in$ $\{0,1\}^{*}, i \in\{1, \ldots, l\}$, any condition $w \in\{0,1\}^{*}$ and any message $m \in\{0,1\}^{k}$, if $(m p k, m s k) \leftarrow \operatorname{Setup}\left(1^{k}\right)$, $s k_{I D} \leftarrow \operatorname{KeyGen}(m s k, I D)$, for all $I D$ used in the system, $r k_{w, I D_{i} \rightarrow I D_{i^{\prime}}} \leftarrow \operatorname{ReKeyGen}\left(I D_{i}, s k_{I D_{i}}, I D_{i^{\prime}}, w\right)$, we have

$$
\begin{aligned}
& \operatorname{Dec}\left(s k_{I D_{1}}, w, C_{1, I D_{1}, w}\right)=m ; \\
& \operatorname{Dec}\left(s k_{I D_{i}}, w, \operatorname{Re} \operatorname{Enc}\left(r k_{w, I D_{i-1} \rightarrow I D_{i}}, w,\right.\right. \\
& \operatorname{ReEnc}\left(r k_{w, I D_{i-2} \rightarrow I D_{i-1}}, w, \ldots,\right. \\
& \left.\left.\left.\operatorname{Re} \operatorname{Enc}\left(r k_{w, I D_{1} \rightarrow I D_{2}}, w, \operatorname{Enc}\left(I D_{1}, w, m\right)\right)\right)\right) \ldots\right)=m .
\end{aligned}
$$

\section{B. Security Models}

We define four security models in terms of the selective condition and selective identity chosen ciphertext security (IND-sCon-sID-CCA), collusion resistance, the anonymity of the original ciphertext and anonymity of the re-encryption key in this section. Before proceeding, we define some notations used in the following definition.

\section{Notations.}

- Delegation Chain. There is a set of re-encryption keys $R K=\left\{r k_{w, I D_{i_{1}} \rightarrow I D_{i_{2}}}, \ldots, r k_{w, I D_{i_{l-1}} \rightarrow I D_{i_{l}}}\right\}$ under the same condition $w$, for any re-encryption key $r k_{w, I D_{i_{j}} \rightarrow I D_{i_{j+1}}}$ in $R K, I D_{i_{j}} \neq I D_{i_{j+1}}$. We say that there exists a delegation chain under $w$ from identity $I D_{i_{1}}$ to identity $I D_{i_{l}}$, denoted as $w \mid I D_{i_{1}} \rightarrow \ldots \rightarrow I D_{i_{l}}$. Note this delegation chain includes the case where $I D_{i_{1}}=$ $I D_{i_{l}}$. Besides, we can use $w \mid I D$ to indicate a ciphertext under $w$ and $I D$, and for a single identity $I D$ we can use $\perp \mid I D$ to denote it.

- Uncorrupted/Corrupted Identity. If the secret key of an identity is compromised by an adversary, the identity is a corrupted identity. Otherwise, it is an uncorrupted identity.

- Uncorrupted Delegation Chain. Suppose there is a delegation chain under $w$ from $I D_{i}$ to $I D_{j}$ (i.e. $w \mid I D_{i} \rightarrow$ $\ldots \rightarrow I D_{j}$ ). If there is no corrupted identity in the chain, it is an uncorrupted delegation chain. Otherwise, it is a corrupted one.

Definition 2: A unidirectional MH-IBCPRE scheme is IND-sCon-sID-CCA-secure if no PPT adversary $\mathcal{A}$ can win the game below with non-negligible advantage. In the game, $\mathcal{B}$ is the game challenger and $k$ is the security parameter.

1) Init. $\mathcal{A}$ outputs a challenge identity $I D^{*} \in\{0,1\}^{*}$ and a challenge condition $w \in\{0,1\}^{*}$.

2) Setup. $\mathcal{B}$ runs $\operatorname{setup}\left(1^{k}\right)$ and returns $m p k$ to $\mathcal{A}$.

3) Phase 1. $\mathcal{A}$ is given access to the following oracles.

a) $\mathcal{O}_{s k}(I D)$ : given an identity $I D$, output $s k_{I D} \leftarrow$ $\operatorname{KeyGen}(m s k, I D)$. b) $\mathcal{O}_{r k}\left(I D_{i}, I D_{i^{\prime}}, w\right)$ : on input two distinct identities $I D_{i}$ and $I D_{i^{\prime}}$, and a condition $w$, output $r k_{w, I D_{i} \rightarrow I D_{i^{\prime}}} \leftarrow \operatorname{ReKeyGen}\left(I D_{i}, s k_{I D_{i}}, I D_{i^{\prime}}, w\right)$, where $s k_{I D_{i}} \leftarrow \operatorname{KeyGen}\left(m s k, I D_{i}\right)$.

c) $\mathcal{O}_{r e}\left(I D_{i}, I D_{i^{\prime}}, w, C_{l, I D_{i}, w}\right)$ : on input two distinct identities $I D_{i}$ and $I D_{i^{\prime}}$, a condition $w$, and an $l$ level ciphertext $C_{l, I D_{i}, w}$ under $I D_{i}$ and $w$, output $C_{l+1, I D_{i}, w} \leftarrow \operatorname{Re} \operatorname{Enc}\left(r k_{w, I D_{i} \rightarrow I D_{i^{\prime}}}, w, C_{l, I D_{i}, w}\right)$, where $r k_{w, I D_{i} \rightarrow I D_{i^{\prime}}} \leftarrow \operatorname{ReKeyGen}\left(I D_{i}, s k_{I D_{i}}\right.$, $\left.I D_{i^{\prime}}, w\right), s k_{I D_{i}} \leftarrow \operatorname{KeyGen}\left(m s k, I D_{i}\right)$.

d) $\mathcal{O}_{\text {dec }}\left(I D_{i}, w, C_{l, I D_{i}, w}\right)$ : on input an identity $I D_{i}$, a condition $w$, and an $l$-level ciphertext $C_{l, I D_{i}, w}$, output $m \leftarrow \operatorname{Dec}\left(s k_{I D_{i}}, w, C_{l, I D_{i}, w}\right)$, where $s k_{I D_{i}} \leftarrow$ $\operatorname{KeyGen}\left(m s k, I D_{i}\right)$.

In this phase the followings are forbidden to issue:

- $\mathcal{O}_{s k}(I D)$ for any $I D$, if there is an uncorrupted delegation chain under $w^{*}$ from $I D^{*}$ to $I D$, or $I D^{*}=I D$.

- $\mathcal{O}_{r k}\left(I D_{i}, I D_{i^{\prime}}, w^{*}\right)$ for any $I D_{i}, I D_{i^{\prime}}$, if there is an uncorrupted delegation chain under $w^{*}$ from $I D^{*}$ to $I D_{i}$ or $I D^{*}=I D_{i}$, but $I D_{i^{\prime}}$ is in a corrupted delegation chain.

4) Challenge. $\mathcal{A}$ outputs two equal length messages $m_{0}, m_{1}$, and a set of identities $\left\{I D_{i_{j}}\right\}_{j=1}^{j=l^{*}-1}$ to $\mathcal{B}$. $\mathcal{B}$ computes $C_{l^{*}, I D^{*}, w^{*}}$ as

$$
\begin{aligned}
& \operatorname{ReEnc}\left(\operatorname{ReKeyGen}\left(I D_{i_{l^{*}-1}}, s k_{I D_{i_{l^{*}-1}}}, I D^{*}, w^{*}\right),\right. \\
& \operatorname{ReEnc}\left(\operatorname{ReKeyGen}\left(I D_{i_{l^{*}-2}}, s k_{I D_{i_{l^{*}-}}}, I D_{i_{l^{*}-1}}, w^{*}\right),\right. \\
& \quad \ldots, \operatorname{ReEnc}\left(\operatorname{ReKeyGen}\left(I D_{i_{1}}, s k_{I D_{i_{1}}}, I D_{i_{2}}, w^{*}\right),\right. \\
& \left.\left.\left.\operatorname{Enc}\left(I D_{i_{1}}, w^{*}, m_{b}\right)\right)\right)\right)
\end{aligned}
$$

where $l^{*} \geq 2, l^{*} \in \mathbb{N}, b \in_{R}\{0,1\}$. Note that we here put $I D^{*}$ to the $l^{*}$ level of the ciphertext. This shows no difference from putting it in the first level of the ciphertext since the system supports multi-hop property.

5) Phase 2. Same as in Phase 1 except the followings:

a) $\mathcal{O}_{r e}\left(I D_{i}, I D_{i^{\prime}}, w^{*}, C_{l, I D_{i}, w^{*}}\right)$ : if $\left(I D_{i}, C_{l, I D_{i}, w^{*}}\right)$ is a derivative of $\left(I D^{*}, C_{l^{*}, I D^{*}, w^{*}}\right)$, and $I D_{i^{\prime}}$ is in a corrupted delegation chain. As of [11], a derivative of $\left(I D^{*}, C_{l^{*}, I D^{*}, w^{*}}\right)$ is defined as follows.

i. $\left(I D^{*}, C_{l^{*}, I D^{*}, w^{*}}\right)$ is a derivative of itself.

ii. If $\left(I D_{i}, C_{l, I D_{i}, w^{*}}\right)$ is a derivative of $\left(I D^{*}, C_{l^{*}, I D^{*}, w^{*}}\right), \quad$ and $\quad\left(I D_{i^{\prime}}, C_{l^{\prime}, I D_{i^{\prime}}, w^{*}}\right)$ is a derivative of $\left(I D_{i}, C_{l, I D_{i}, w^{*}}\right)$, then $\left(I D_{i^{\prime}}, C_{l^{\prime}, I D_{i^{\prime}}, w^{*}}\right)$ is a derivative of $\left(I D^{*}, C_{l^{*}, I D^{*}, w^{*}}\right)$, where $l^{\prime} \geq l \geq l^{*}$.

iii. If $\mathcal{A}$ has issued a re-encryption key query to $\mathcal{O}_{r k}$ on $\left(I D_{i}, I D_{i^{\prime}}, w\right)$ to obtain the re-encryption key $r k_{w, I D_{i} \rightarrow I D_{i^{\prime}}}$, and achieved $C_{\left(l+1, I D_{i^{\prime}}, w\right)} \leftarrow$ $\operatorname{ReEnc}\left(r k_{w, I D_{i} \rightarrow I D_{i^{\prime}}}, w, C_{\left(l, I D_{i}, w\right)}\right)$, then $\left(I D_{i^{\prime}}\right.$, $\left.C_{\left(l+1, I D_{i^{\prime}}, w\right)}\right)$ is a derivative of $\left(I D_{i}, C_{\left(l, I D_{i}, w\right)}\right)$.

iv. If $\mathcal{A}$ can execute $C_{\left(l+1, I D_{i^{\prime}}, w\right)} \leftarrow$ $\operatorname{ReEnc}\left(\operatorname{ReKeyGen}\left(I D_{i}, s_{I D_{i}}, I D_{i^{\prime}}, w\right), w\right.$, $\left.C_{\left(l, I D_{i}, w\right)}\right)$ on its own, then $\left(I D_{i^{\prime}}, C_{\left(l+1, I D_{i^{\prime}}, w\right)}\right)$ is a derivative of $\left(I D_{i}, C_{\left(l, I D_{i}, w\right)}\right)$, where $s k_{I D_{i}} \leftarrow \operatorname{KeyGen}\left(m s k, I D_{i}\right)$. 
v. If $\mathcal{A}$ has issued a re-encryption query on $\left(I D_{i}, I D_{i^{\prime}}, w, C_{\left(l, I D_{i}, w\right)}\right) \quad$ and obtained $C_{\left(l+1, I D_{i^{\prime}}, w\right)}$, then $\left(I D_{i^{\prime}}, C_{\left(l+1, I D_{i^{\prime}}, w\right)}\right)$ is a derivative of $\left(I D_{i}, C_{\left(l, I D_{i}, w\right)}\right)$.

b) $\mathcal{O}_{d e c}\left(I D_{i}, w^{*}, C_{l, I D_{i}, w^{*}}\right)$ : if $\left(I D_{i}, C_{l, I D_{i}, w^{*}}\right)$ is a derivative of $\left(I D^{*}, C_{l^{*}, I D^{*}, w^{*}}\right)$.

6) Guess. $\mathcal{A}$ outputs a guess $b^{\prime} \in\{0,1\}$. If $b^{\prime}=b, \mathcal{A}$ wins.

The advantage of $\mathcal{A}$ is defined as $\epsilon=$ $A d v_{M H-I B C P R E, \mathcal{A}}^{I N D-s C o n-s I D-C C A}\left(1^{k}\right)=\left|\operatorname{Pr}\left[b^{\prime}=b\right]-\frac{1}{2}\right|$.

We now proceed to collusion resistance. This property guarantees that an adversary cannot compromise the entire secret key of a delegator even if it colludes with the delegatee.

Definition 3: A unidirectional MH-IBCPRE scheme holds against selective collusion attacks if the advantage $A d v_{\mathcal{A}}^{C R}\left(1^{k}\right)$ is negligible for any PPT adversary $\mathcal{A}$ in the following experiment. Set $O_{1}=\left\{\mathcal{O}_{s k}, \mathcal{O}_{r k}\right\}$ and $A d v_{\mathcal{A}}^{C R}\left(1^{k}\right)$ as

$$
\begin{aligned}
& \operatorname{Pr}\left[s k_{I D^{*}} \in \Omega:\left(I D^{*}, \text { State }\right) \leftarrow \mathcal{A}\left(1^{k}\right) ;\right. \\
& \left.(m p k, m s k) \leftarrow \operatorname{Setup}\left(1^{k}\right) ; s k_{I D^{*}} \leftarrow \mathcal{A}^{O_{1}}(\text { mpk }, \text { State })\right]
\end{aligned}
$$

where $k$ is the security parameter, State is the state information, $I D^{*}$ is the target and uncorrupted identity, $\mathcal{O}_{s k}$ and $\mathcal{O}_{r k}$ are the oracles defined in Definition 2, $\Omega$ is the valid secret key space, and $s k_{I D^{*}}$ is the valid secret key of $I D^{*}$. If $\mathcal{A}$ issues $I D^{*}$ to $\mathcal{O}_{s k}$, output $\perp$.

Below we define the anonymity of the original ciphertext (ANO-OC) for MH-IBCPRE, that is, given the original ciphertext, an adversary cannot tell the identity of delegator.

Definition 4: A unidirectional MH-IBCPRE scheme achieves ANO-OC if the advantage $A d v_{\mathcal{A}}^{A N O-O C}\left(1^{k}\right)$ is negligible for any PPT adversary $\mathcal{A}$ in the following experiment. Set $O_{2}=\left\{\mathcal{O}_{s k}, \mathcal{O}_{r k}, \mathcal{O}_{r e}, \mathcal{O}_{d e c}\right\}$, and $A d v_{\mathcal{A}}^{A N O-O C}\left(1^{k}\right)$ as

$$
\begin{aligned}
& \mid \operatorname{Pr}\left[b=b^{\prime}:\left(w^{*}, I D_{0}^{*}, \text { ID }_{1}^{*}, \text { State }_{1}\right) \leftarrow \mathcal{A}\left(1^{k}\right) ;(\text { mpk },\right. \\
& \text { msk }) \leftarrow \text { Setup }\left(1^{k}\right) ;\left(m, \text { State }_{2}\right) \leftarrow A^{O_{2}}\left(\text { mpk }, \text { State }_{1}\right) ; \\
& b \in_{R}\{0,1\} ; C_{1, I D_{b}^{*}, w^{*}} \leftarrow \operatorname{Enc}\left(I D_{b}^{*}, w^{*}, m\right) ; \\
& \left.b^{\prime} \leftarrow A^{O_{2}}\left(C_{1, I D_{b}^{*}, w^{*}}, \text { State }_{2}\right) ;\right]-\frac{1}{2} \mid
\end{aligned}
$$

where $k$ is the security parameter, State $_{1}$, State $_{2}$ are the state information, $I D_{0}^{*}, I D_{1}^{*}$ are two distinct uncorrupted identities, $C_{1, I D_{b}^{*}, w^{*}}$ is constructed by the game challenger, $\mathcal{O}_{s k}, \mathcal{O}_{r k}$, $\mathcal{O}_{r e}, \mathcal{O}_{d e c}$ are the oracles with the following constraints. In $\mathcal{O}_{s k}$, the oracle outputs $\perp$ if there is an uncorrupted delegation chain under $w^{*}$ from $I D_{b}^{*}$ to $I D$ or $I D_{b}^{*}=I D$. In $\mathcal{O}_{r k}$, the oracle outputs $\perp$ if there is an uncorrupted delegation chain under $w^{*}$ from $I D_{b}^{*}$ to $I D_{i}$ or $I D_{b}^{*}=I D_{i}$, and $I D_{i^{\prime}}$ is in a corrupted delegation chain. For $\mathcal{O}_{r e}$, if the issued ciphertext is a derivative of $\left(I D_{b}^{*}, C_{1, I D_{b}^{*}, w^{*}}\right)$, and $I D_{i^{\prime}}$ is in a corrupted delegation chain, output $\perp$. For $\mathcal{O}_{d e c}$, if the issued ciphertext is a derivative of $\left(I D_{b}^{*}, C_{1, I D_{b}^{*}, w^{*}}\right)$, output $\perp$.

Finally, we define the anonymity of the re-encryption key (ANO-RK), in which an adversary cannot distinguish a real re-encryption key from a random one.
Definition 5: A unidirectional MH-IBCPRE scheme achieves ANO-RK if no PPT adversary $\mathcal{A}$ can win the game below with non-negligible advantage.

1) Init. $\mathcal{A}$ outputs a delegator's identity $I D^{\prime}$, a challenge delegatee's identity $I D^{*}$, and a challenge condition $w^{*}$.

2) Setup. Same as Definition 2.

3) Phase 1. $\mathcal{A}$ is allowed to issue queries to $\mathcal{O}_{s k}, \mathcal{O}_{r k}, \mathcal{O}_{r e}$ and $\mathcal{O}_{d e c}$ which are the oracles defined Definition 2 with the same restrictions.

4) Challenge. If the following queries

- $\mathcal{O}_{s k}\left(I D_{i}\right)$ for any $I D_{i}$, if there is an uncorrupted delegation chain under $w^{*}$ from $I D^{*}$ to $I D_{i}$, or $I D^{*}=I D_{i}$.

- $\mathcal{O}_{r k}\left(I D_{i}, I D_{j}, w^{*}\right)$ for any $I D_{i}, I D_{j}$, if there is an uncorrupted delegation chain under $w^{*}$ from $I D^{*}$ to $I D_{i}$ or $I D^{*}=I D_{i}$, but $I D_{j}$ is in a corrupted delegation chain.

are never made, $\mathcal{B}$ flips a coin-toss for $b \in\{0,1\}$. Then $\mathcal{B}$ sets the re-encryption key $r k_{w^{*}, I D^{\prime} \rightarrow I D^{*}}$ as a random key from the re-encryption key space if $b=0$ and computes $r k_{w^{*}, I D^{\prime} \rightarrow I D^{*}} \leftarrow \operatorname{ReKeyGen}\left(I D^{\prime}, s k_{I D^{\prime}}, I D^{*}, w^{*}\right)$ otherwise. Finally, $\mathcal{B}$ outputs $r k_{w^{*}, I D^{\prime} \rightarrow I D^{*}}$ to $\mathcal{A}$.

5) Phase 2. Same as Phase 1 except the followings:

a) $\mathcal{O}_{s k}\left(I D_{i}\right)$ for any $I D_{i}$, if there is an uncorrupted delegation chain under $w^{*}$ from $I D^{*}$ to $I D_{i}$, or $I D^{*}=I D_{i}$

b) $\mathcal{O}_{r k}\left(I D_{i}, I D_{j}, w^{*}\right)$ for any $I D_{i}, I D_{j}$, if there is an uncorrupted delegation chain under $w^{*}$ from $I D^{*}$ to $I D_{i}$ or $I D^{*}=I D_{i}$, but $I D_{j}$ is in a corrupted delegation chain;

c) $\mathcal{O}_{r e}\left(I D_{i}, I D_{i^{\prime}}, w^{*}, C_{l, I D_{i}, w^{*}}\right)$ : if $\left(I D_{i}, C_{l, I D_{i}, w^{*}}\right)$ is a (derivative of) ciphertext generated by a re-encryption key in the delegation chain under $w^{*}$ from $I D^{*}$ to $I D_{i}$, and $I D_{i^{\prime}}$ is in a corrupted delegation chain; and

d) $\mathcal{O}_{\text {dec }}\left(I D_{i}, w^{*}, C_{l, I D_{i}, w^{*}}\right)$ : if $\left(I D_{i}, C_{l, I D_{i}, w^{*}}\right)$ is a (derivative of) ciphertext generated by a re-encryption key in the delegation chain under $w^{*}$ from $I D^{*}$ to $I D_{i}$.

6) Guess. $\mathcal{A}$ outputs a guess $b^{\prime} \in\{0,1\}$. If $b^{\prime}=b, \mathcal{A}$ wins. The advantage of $\mathcal{A}$ is defined as $\operatorname{Adv} v_{\mathcal{A}}^{A N O-R K}\left(1^{k}\right)=$ $\left|\operatorname{Pr}\left[b^{\prime}=b\right]-\frac{1}{2}\right|$.

Remark. As sated in [1], the anonymity of the re-encrypted ciphertext is implied by the anonymity of the re-encryption key, we hence omit the details here. We refer the reader to [1] for more details.

\section{Complexity Assumptions And Building Blocks}

\section{A. Asymmetric Pairings}

Let BSetup be an algorithm that on input the security parameter $k$, outputs the parameters of a bilinear map as $\left(q, g, \hat{g}, \mathbb{G}_{1}, \mathbb{G}_{2}, \mathbb{G}_{T}, e\right)$, where $\mathbb{G}_{1}, \mathbb{G}_{2}$ and $\mathbb{G}_{T}$ are multiplicative cyclic groups of prime order $q$, where $|q|=k$, and $g$ is a random generator of $\mathbb{G}_{1}, \hat{g}$ is a random generator of $\mathbb{G}_{2}$. The mapping $e: \mathbb{G}_{1} \times \mathbb{G}_{2} \rightarrow \mathbb{G}_{T}$ has three properties: (1) Bilinearity: for all $a, b \in_{R} \mathbb{Z}_{q}^{*}, e\left(g^{a}, \hat{g}^{b}\right)=e(g, \hat{g})^{a b}$; (2) Nondegeneracy: $e(g, \hat{g}) \neq 1_{\mathbb{G}_{T}}$, where $1_{\mathbb{G}_{T}}$ is the unit of $\mathbb{G}_{T}$; (3) Computability: $e$ can be efficiently computed. Note that $G_{1}$ and $G_{2}$ are not the same. 


\section{B. Complexity Assumptions}

Asymmetric Bilinear Diffie-Hellman (ABDH) Problem [14]. Given a tuple $\left(g, g^{a}, g^{c}, \hat{g}, \hat{g}^{a}, \hat{g}^{b}\right) \in \mathbb{G}_{1}^{3} \times \mathbb{G}_{2}^{3}$, compute $e(g, \hat{g})^{a b c} \in \mathbb{G}_{T}$.

Asymmetric Decisional BDH (ADBDH) Problem [14]. Given a tuple $\left(g, g^{a}, g^{c}, \hat{g}, \hat{g}^{a}, \hat{g}^{b}\right) \in \mathbb{G}_{1}^{3} \times \mathbb{G}_{2}^{3}$ and $T \in \mathbb{G}_{T}$, decide whether $T=e(g, \hat{g})^{a b c}$.

(Asymmetric) Decisional $\mathcal{P}$-BDH Problem [14]. Given a tuple $\left(g, g^{a}, g^{a b}, g^{c}, \hat{g}, \hat{g}^{a}, \hat{g}^{b}\right) \in \mathbb{G}_{1}^{4} \times \mathbb{G}_{2}^{3}$ and $T \in \mathbb{G}_{T}$, decide whether $T=e(g, \hat{g})^{a b c}$.

Definition 6: ADBDH Assumption [14]. We say that an algorithm $\mathcal{A}$ has advantage $A d v_{\mathcal{A}}^{A D B D H}=\epsilon$ in solving the $A D B D H$ problem in $\left(\mathbb{G}_{1}, \mathbb{G}_{2}\right)$ if $\mid \operatorname{Pr}\left[\mathcal{A}\left(g, g^{a}, g^{c}, \hat{g}, \hat{g}^{a}, \hat{g}^{b}, e(g, \hat{g})^{a b c}\right)=0\right]-$ $\operatorname{Pr}\left[\mathcal{A}\left(g, g^{a}, g^{c}, \hat{g}, \hat{g}^{a}, \hat{g}^{b}, T\right)=0\right] \mid \geq \epsilon$, where the probability is over the random choice of generators $g \in \mathbb{G}_{1}$ and $\hat{g} \in \mathbb{G}_{2}$, the random choice of exponents $a, b, c \in \mathbb{Z}_{q}^{*}, T \in \mathbb{G}_{T}$, and the random bits used by $\mathcal{A}$. We say that the ADBDH assumption holds in $\left(\mathbb{G}_{1}, \mathbb{G}_{2}\right)$ if no PPT algorithm has advantage $\epsilon$ in solving the ASBDH problem in $\left(\mathbb{G}_{1}, \mathbb{G}_{2}\right)$.

Definition 7: (Asymmetric) Decisional $\mathcal{P}$-BDH Assumption [14]. We say that an algorithm $\mathcal{A}$ has advantage $A d v_{\mathcal{A}}^{\mathcal{P}-B D H}=\epsilon$ in solving the decisional $\mathcal{P}-B D H$ problem in $\left(\mathbb{G}_{1}, \mathbb{G}_{2}\right)$ if $\mid \operatorname{Pr}\left[\mathcal{A}\left(g, g^{a}, g^{a b}, g^{c}, \hat{g}, \hat{g}^{a}, \hat{g}^{b}, g^{a b c}\right)=0\right]-$ $\operatorname{Pr}\left[\mathcal{A}\left(g, g^{a}, g^{a b}, g^{c}, \hat{g}, \hat{g}^{a}, \hat{g}^{b}, T\right)=0\right] \mid \geq \epsilon$, where the probability is over the random choice of generators $g \in \mathbb{G}_{1}$ and $\hat{g} \in \mathbb{G}_{2}$, the random choice of exponents $a, b, c \in \mathbb{Z}_{q}^{*}, T \in \mathbb{G}_{1}$, and the random bits used by $\mathcal{A}$. We say that the decisional $\mathcal{P}$-BDH assumption holds in $\left(\mathbb{G}_{1}, \mathbb{G}_{2}\right)$ if no PPT algorithm has advantage $\epsilon$ in solving the decisional $\mathcal{P}$-BDH problem in $\left(\mathbb{G}_{1}, \mathbb{G}_{2}\right)$.

\section{Strongly Existential Unforgeable One-Time Signatures.}

A strongly existential unforgeable (sUF) one-time signature [4] consists of the following algorithms:

1) $\left(K_{s}, K_{v}\right) \leftarrow \operatorname{Sig} . K G\left(1^{k}\right)$ : on input a security parameter $k \in \mathbb{N}$, the algorithm outputs a signing/ verification key pair $\left(K_{s}, K_{v}\right)$.

2) $\sigma \leftarrow \operatorname{Sign}\left(K_{s}, M\right)$ : on input the signing key $K_{s}$ and a message $M \in \Gamma_{\text {Sig }}$, the algorithm outputs a signature $\sigma$, where $\Gamma_{S i g}$ is the message space of a signature scheme.

3) $1 / 0 \leftarrow \operatorname{Ver}\left(K_{v}, \sigma, M\right)$ : on input the verification key $K_{v}$, a signature $\sigma$ and a message $M$, the algorithm outputs 1 when $\sigma$ is a valid signature of $M$, and output 0 otherwise.

Definition 8: A signature scheme is one-time strongly unforgeable chosen-message attack (sUF-CMA) secure if the advantage $A d v_{\mathcal{A}}^{s U F-C M A}\left(1^{k}\right)$ is negligible for any PPT adversary $\mathcal{A}$ in the following experiment.

$$
\begin{aligned}
& A d v_{\mathcal{A}}^{s U F-C M A}\left(1^{\lambda}\right)=\operatorname{Pr}\left[\operatorname{Ver}\left(K_{v}, \sigma^{*}, M^{*}\right)=1:\right. \\
& \left(K_{s}, K_{v}\right) \leftarrow \operatorname{Sig} \cdot K G\left(1^{k}\right) ;(M, \text { State }) \leftarrow \mathcal{A}\left(K_{v}\right) ; \\
& \sigma \leftarrow \operatorname{Sign}\left(K_{s}, M\right) ;\left(M^{*}, \sigma^{*}\right) \leftarrow \mathcal{A}\left(K_{v}, \sigma, \text { State }\right) ; \\
& \left.\left(M^{*}, \sigma^{*}\right) \neq(M, \sigma)\right],
\end{aligned}
$$

where State is the state information.

\section{One-time Symmetric Encryption}

A one-time symmetric encryption [13] consists of the following algorithms. Note let $\mathcal{K}_{D}$ be the key space $\{0,1\}^{\text {poly }\left(1^{k}\right)}$, and $S Y M$ be a symmetric encryption scheme, where $\operatorname{poly}\left(1^{k}\right)$ is the fixed polynomial size (bound) with respect to the security parameter $k$. The encryption algorithm $S Y M$.Enc intakes a key $K \in \mathcal{K}_{D}$ and a message $M$, outputs a ciphertext $C$. The decryption algorithm $S Y M$.Dec intakes $K$ and $C$, outputs $M$ or a symbol $\perp$.

\section{E. Target Collision Resistant Hash Function.}

Target Collision Resistant (TCR) hash function was introduced by Cramer and Shoup [13]. A TCR hash function $H$ guarantees that given a random element $x$ which is from the valid domain of $H$, a PPT adversary $\mathcal{A}$ cannot find $y \neq x$ such that $H(x)=H(y)$. We let $A d v_{H, \mathcal{A}}^{T C R}=\operatorname{Pr}[H(x)=$ $H(y) \wedge x \neq y \mid x, y \in D H]$ be the advantage of $\mathcal{A}$ in successfully finding collisions from a TCR hash function $H$, where $D H$ is the valid input domain of $H$. If a hash function is chosen from a TCR hash function family, $A d v_{H, \mathcal{A}}^{T C R}$ is negligible.

\section{F. An Anonymous IBE and Its Extensions}

Ducas [14] introduces an efficient anonymous IBE (DuANO-IBE) scheme in the standard model. We review its construction below, and omit the definition and security model of Du-ANO-IBE as the details can be found in [14].

- $\operatorname{Setup}\left(1^{k}\right): \operatorname{run}\left(q, g, \hat{g}, \mathbb{G}_{1}, \mathbb{G}_{2}, \mathbb{G}_{T}, e\right) \leftarrow \operatorname{BSetup}\left(1^{k}\right)$, choose random values $\alpha, \beta, \gamma, \delta, \eta \in \mathbb{Z}_{q}^{*}$, and set $g_{1}=g^{\alpha}$, $g_{2}=g^{\beta}, h=g^{\gamma}, f=g^{\delta}, t=g^{\eta}, \hat{g_{1}}=\hat{g}^{\alpha}, \hat{g_{2}}=$ $\hat{g}^{\beta}, \hat{h}=\hat{g}^{\gamma}, \hat{f}=\hat{g}^{\delta}, \hat{t}=\hat{g}^{\eta}$. The master secret key $m s k=\left(\hat{g}_{0}=\hat{g}^{\alpha \beta}, \hat{f}, \hat{t}\right)$, the master public key $m p k=$ $\left(g, \hat{g}, g_{1}, h, f, t, \hat{g_{2}}, \hat{h}\right)$.

- Extract $(m s k, I D)$ : given $m s k$ and an identity $I D \in$ $\mathbb{Z}_{q}^{*}$, randomly choose $r, R \in \mathbb{Z}_{q}^{*}$, output $s k_{I D}=$ $\left(s k_{I D_{0}}, s k_{I D_{1}}, s k_{I D_{2}}\right)=\left(\hat{g}_{0}\left(\hat{h}^{I D} \hat{f}\right)^{r} \hat{t}^{R}, \hat{g}^{r}, \hat{g}^{R}\right)$.

- $\operatorname{Enc}(m p k, I D, m)$ : randomly choose $s \in \mathbb{Z}_{q}^{*}$, compute $C_{1}=e\left(g_{1}, \hat{g}_{2}\right)^{s} \cdot m, C_{2}=g^{s}, C_{3}=\left(h^{I D} f\right)^{s}, C_{4}=t^{s}$, and output the ciphertext $C=\left(C_{1}, C_{2}, C_{3}, C_{4}\right)$, where $I D \in \mathbb{Z}_{q}^{*}, m \in \mathbb{G}_{T}$.

- $\operatorname{Dec}\left(s k_{I D}, C\right)$ : given a ciphertext $C=\left(C_{1}, C_{2}, C_{3}, C_{4}\right)$, using the private key $s k_{I D}$ to recover the plaintext $m=$ $C_{1} \cdot e\left(C_{3}, s k_{I D_{1}}\right) \cdot e\left(C_{4}, s k_{I D_{2}}\right) / e\left(C_{2}, s k_{I D_{0}}\right)$.

By Theorem 1 and its corresponding security proof in [14], we have the following theorem.

Theorem 1: Du-ANO-IBE is selective-ID (sID) anonymous and secure against chosen-plaintext attacks assuming the decisional $\mathcal{P}$-BDH assumption holds.

Below we employ the BB1 HIBE technique [6] to extend Du-ANO-IBE to be a two levels encryption scheme without losing CPA security, where the first level is the identity, and the second level is the condition. We state that the first level is anonymous, but the second level is not.

- $\operatorname{Setup}\left(1^{k}\right)$ : let $w \in \mathbb{Z}_{q}^{*}$ be a condition, and choose $\alpha, \beta$, $\gamma, \delta_{1}, \delta_{2}, \eta \in_{R} \mathbb{Z}_{q}^{*}$, and set $g_{1}=g^{\alpha}, g_{2}=g^{\beta}, h=g^{\gamma}$, $f_{1}=g^{\delta_{1}}, f_{2}=g^{\delta_{2}}, t=g^{\eta}, \hat{g}_{1}=\hat{g}^{\alpha}, \hat{g}_{2}=\hat{g}^{\beta}, \hat{h}=\hat{g}^{\gamma}$, 
$\hat{f}_{1}=\hat{g}^{\delta_{1}}, \hat{f}_{2}=\hat{g}^{\delta_{2}}, \hat{t}=\hat{g}^{\eta}$. The master secret key $m s k=\left(\hat{g}_{0}=\hat{g}^{\alpha \beta}, \hat{f}_{1}, \hat{t}\right)$, the master public key $m p k=$ $\left(g, \hat{g}, g_{1}, h, f_{1}, f_{2}, t, \hat{g}_{2}, \hat{f}_{2}, \hat{h}\right)$.

- $\operatorname{Extract}(m s k, I D)$ : set $s k_{I D}=\left(s k_{I D_{0}}, s k_{I D_{1}}, s k_{I D_{2}}\right.$, $\left.s k_{I D_{3}}\right)=\left(\hat{g}_{0}\left(\hat{h}^{I D} \hat{f}_{1}\right)^{r_{1}}\left(\hat{h}^{w} \hat{f}_{2}\right)^{r_{2}} \hat{t}^{R}, \hat{g}^{r_{1}}, \hat{g}^{r_{2}}, \hat{g}^{R}\right)$, where $r_{1}, r_{2}, R \in \mathbb{Z}_{q}^{*}$. Given $s k_{I D}=\left(s k_{I D_{0}}, s k_{I D_{1}}, s k_{I D_{2}}\right)$ which is generated in the algorithm Extract of DuANO-IBE, one can easily derive the above secret key by using the BB1 construction technique. To achieve the consistency of algorithm description, we here use the "same" secret key generation expression.

- $\operatorname{Enc}(m p k, I D, m, w)$ : set $C_{1}=e\left(g_{1}, \hat{g}_{2}\right)^{s} \cdot m, C_{2}=g^{s}$, $C_{3}=\left(h^{I D} f_{1}\right)^{s}, C_{4}=t^{s}$ and $C_{5}=\left(h^{w} f_{2}\right)^{s}$, where $s \in \mathbb{Z}_{q}^{*}$.

- $\operatorname{Dec}\left(s k_{I D}, C\right)$ : compute $m=C_{1} \cdot e\left(C_{3}, s k_{I D_{1}}\right)$. $e\left(C_{5}, s k_{I D_{2}}\right) \cdot e\left(C_{4}, s k_{I D_{3}}\right) / e\left(C_{2}, s k_{I D_{0}}\right)$.

We refer to the above system as 2-level Du-ANO-HIBE. As stated in [14], Du-ANO-IBE can be extended to 2-level system to achieve CCA security via BB1 HIBE construction technique. The above system is exactly the converted 2-level system except that the second level is a condition instead of a verification key (of a one-time signature). Here the CPA security of 2-level Du-ANO-HIBE still relies on the decisional $\mathcal{P}$-BDH assumption, and the corresponding proof is straightforward to reuse the proof technique presented in [14]. Therefore, we have the following theorem.

Theorem 2: 2-level Du-ANO-HIBE is anonymous and CPA secure assuming the decisional $\mathcal{P}$-BDH assumption holds.

\section{G. A CCA-Secure 3-Level Du-ANO-HIBE}

Here we convert 2-level Du-ANO-HIBE to achieve CCA security by using the CHK transformation [10]. Following the BB1 HIBE construction, a 3-level CCA-secure anonymous system, which is anonymous relative to the first level but not the second and third levels, can be built as follows.

- $\operatorname{Setup}\left(1^{k}\right)$ : same as the algorithm Setup of 2-level DuANO-HIBE except the followings. Choose random values $\delta_{3} \in \mathbb{Z}_{q}^{*}$, and set $f_{3}=g^{\delta_{3}}$ and $\hat{f}_{3}=\hat{g}^{\delta_{3}}$. Choose an sUF one-time signature scheme (Sig.KG, Sign, Ver) and set the verification key $K_{v}$ is in $\mathbb{Z}_{q}^{*}$, where $k_{1}$ is the security parameter. The master public key $m p k=\left(g, \hat{g}, g_{1}, h\right.$, $f_{1}, f_{2}, f_{3}, t, \hat{g}_{2}, \hat{f}_{2}, \hat{f}_{3}, \hat{h},($ Sig.KG, Sign, Ver $)$ ).

- Extract $(m s k, I D)$ : set $s k_{I D}=\left(s k_{I D_{0}}, s k_{I D_{1}}, s k_{I D_{2}}\right.$, $\left.s k_{I D_{3}}, s k_{I D_{4}}\right)=\left(\hat{g}_{0}\left(\hat{h}^{I D} \hat{f}_{1}\right)^{r_{1}}\left(\hat{h}^{w} \hat{f}_{2}\right)^{r_{2}}\left(\hat{h}^{K_{v}} \hat{f}_{3}\right)^{r_{3}} \hat{t}^{R}\right.$, $\left.\hat{g}^{r_{1}}, \hat{g}^{r_{2}}, \hat{g}^{r_{3}}, \hat{g}^{R}\right)$, where $r_{1}, r_{2}, r_{3}, R \in \mathbb{Z}_{q}^{*}$. To achieve consistency of description, we keep the secret key generation in one algorithm. Actually, given the secret key of 2-level Du-ANO-HIBE, one can easily derive the above key.

- $\operatorname{Enc}\left(m p k, I D, m, K_{v}\right)$ : choose $s \in_{R} \mathbb{Z}_{q}^{*}$ and a one-time signature key pair $\left(K_{s}, K_{v}\right) \leftarrow \operatorname{Sig} . K G\left(1^{k}\right)$, set $C_{0}=$ $K_{v}, C_{1}=e\left(g_{1}, \hat{g}_{2}\right)^{s} \cdot m, C_{2}=g^{s}, C_{3}=\left(h^{I D} f_{1}\right)^{s}, C_{4}=$ $t^{s}, C_{5}=\left(h^{w} f_{2}\right)^{s}, C_{6}=\left(h^{K_{v}} f_{3}\right)^{s}, C_{7}=\operatorname{Sign}\left(K_{s},\left(C_{1}\right.\right.$, $\left.\left.C_{2}, C_{3}, C_{4}, C_{5}, C_{6}\right)\right)$.

- $\operatorname{Dec}\left(s k_{I D}, C\right)$ : given a ciphertext $C=\left(C_{0}, C_{1}, C_{2}\right.$, $\left.C_{3}, C_{4}, C_{5}, C_{6}, C_{7}\right)$, first verify whether $e\left(\hat{h}^{K_{v}} \hat{f}_{3}\right.$, $\left.C_{2}\right)=e\left(\hat{g}, C_{6}\right)$ and $\operatorname{Ver}\left(K_{v}, C_{7},\left(C_{1}, C_{2}, C_{3}, C_{4}, C_{5}\right.\right.$,
$\left.\left.C_{6}\right)\right)=1$ hold. If the equations do not hold, output $\perp$. Otherwise, compute $m=C_{1} \cdot e\left(C_{3}, s k_{I D_{1}}\right) \cdot e\left(C_{5}, s k_{I D_{2}}\right)$. $e\left(C_{6}, s k_{I D_{3}}\right) \cdot e\left(C_{4}, s k_{I D_{4}}\right) / e\left(C_{2}, s k_{I D_{0}}\right)$.

We refer to the above system as 3-level Du-ANO-HIBE. By Theorem 2 and the security argument in [14], we have the following theorem.

Theorem 3: If 2-level Du-ANO-HIBE is sID anonymous and CPA secure, and (Sig.KG,Sign, Ver) is an sUF onetime signature scheme, 3-level Du-ANO-HIBE is sID anonymous and CCA secure.

The security proof is straight forward to reuse the technique proposed in [14].

\section{A NEW AMH-IBCPRE SCHEME}

We first show the difficulties of our construction, and next present the technical roadmap.

Difficulties in converting existing primitives to achieve our goals. [31] proposed an IBPRE system with the anonymity property. Nonetheless, the system cannot be trivially extended to satisfy the conditional sharing and multiple receivers update. This is because the construction of re-encryption key and re-encrypted ciphertext both limit its extension. In the reencryption key generation, there is no interface for a condition, unless we bond the condition with the identity and further regard them as a whole "identity". But this hinders the flexibility of the system as for each distinct condition, the private key generator has to generate a private key for user accordingly. The number of private key now increases to $O(a b)$, where $a$ is the maximum number of condition belonging to a user, and $b$ is the number of system user.

In the re-encryption, the original ciphertext component $c_{1}$ and $c_{2}$ are necessary components. Only $c_{2}$ but not $c_{1}$ is included in re-encrypted ciphertext. This explains why a reencrypted ciphertext holder cannot make a further conversion. If we want to extend the ciphertext to support multiple reencryption, we have to keep both components simultaneously. However, this will make the system insecure in the security of re-encrypted ciphertext as an adversary can leverage them and a re-encryption key from the challenge identity to a corrupted user to re-encrypt the challenge ciphertext to one whose plaintext can be recovered.

In addition to [31], we observe that [32], [24], [22] also proposed useful IBPRE systems, which partially achieve our goals. Nevertheless, none of these systems can be extended to support anonymity. This is because they are built based on non-anonymous IBE systems [39], [7] and [38], respectively. In a non-anonymous IBE, the identity information can be easily checked by pairings computation. Note we here refer reader to [38] for more details.

To achieve all our goals at the same time, we follow the technical roadmap below. We first start with an anonymous IBE [14], and next extend the IBE system to become anonymous 3 levels hierarchical IBE with CCA security by leveraging CHK transformation [10], in which one level is for identity, one is for condition and the rest is for verification key. Based on the resulting system, we build an 
anonymous multi-hop identity-based conditional proxy reencryption (AMH-IBCPRE) scheme. In the scheme, we leverage the private key derivation property of hierarchical IBE to construct a re-encryption key such that the re-encryption key contains a masked "secret key" for identity vector (identity, condition). We state that even an adversary obtains this so-called secret key it can only achieve the re-encryption but not decryption rights of the ciphertexts corresponding to (identity,condition). For the re-encryption, we utilize a one-time symmetric key encryption technique to "wrap" the re-encryption result and the previous level (re-encrypted) ciphertext, so that these elements cannot be reused by the adversary in the next round re-encryption.

We now start describing our new CCA-secure AMHIBCPRE scheme. We allow condition and identities to be arbitrary length, but they should be hashed by a TCR hash function $H_{0}:\{0,1\}^{*} \rightarrow \mathbb{Z}_{q}^{*}$ beforehand.

1) $\operatorname{Setup}\left(1^{k}\right)$. Given $k$, run $\left(q, g, \hat{g}, \mathbb{G}_{1}, \mathbb{G}_{2}, \mathbb{G}_{T}, e\right) \leftarrow$ $\operatorname{BSetup}\left(1^{k}\right)$. Let $w \in \mathbb{Z}_{q}^{*}$ be a condition. Choose $\alpha, \beta$, $\gamma, \delta_{1}, \delta_{2}, \delta_{3}, \eta \in_{R} \mathbb{Z}_{q}^{*}$, and set $g_{1}=g^{\alpha}, g_{2}=g^{\beta}$, $h=g^{\gamma}, f_{1}=g^{\delta_{1}}, f_{2}=g^{\delta_{2}}, f_{3}=g^{\delta_{3}}, t=g^{\eta}, \hat{g}_{1}=\hat{g}^{\alpha}$, $\hat{g}_{2}=\hat{g}^{\beta}, \hat{h}=\hat{g}^{\gamma}, \hat{f}_{1}=\hat{g}^{\delta_{1}}, \hat{f}_{2}=\hat{g}^{\delta_{2}}, \hat{f}_{3}=\hat{g}^{\delta_{3}}, \hat{t}=\hat{g}^{\eta}$. Choose two TCR hash functions: $H_{1}:\{0,1\}^{k} \rightarrow \mathbb{Z}_{q}^{*}$, $H_{2}: \mathbb{G}_{T} \rightarrow\{0,1\}^{\text {poly }\left(1^{k}\right)}$, and a CCA-secure onetime symmetric key encryption $S Y M=$ (SYM.Enc, SYM.Dec). Let (Sig.KG,Sign, Ver) be a sUF onetime signature scheme and assume any verification key $K_{v}$ in $\mathbb{Z}_{q}^{*}$. The master secret key $m s k=\left(\hat{g}_{0}=\right.$ $\left.\hat{g}^{\alpha \beta}, \hat{f}_{1}, \hat{t}\right)$, the master public key $m p k=(q, k, g, \hat{g}$, $\mathbb{G}_{1}, \mathbb{G}_{2}, \mathbb{G}_{T}, e, g_{1}, h, f_{1}, f_{2}, f_{3}, t, \hat{g}_{2}, \hat{f}_{2}, \hat{f}_{3}, \hat{h}, H_{1}$, $\mathrm{H}_{2}, S Y M,($ Sig.KG, Sign, Ver $\left.)\right)$.

2) Extract $(m s k, I D)$. Given $m s k$ and an identity $I D \in$ $\mathbb{Z}_{q}^{*}$, choose $r, R \in \in_{R} \mathbb{Z}_{q}^{*}$, output $s k_{I D}=\left(s k_{I D_{0}}, s k_{I D_{1}}\right.$, $\left.s k_{I D_{2}}\right)=\left(\hat{g}_{0}\left(\hat{h}^{I D} \hat{f}_{1}\right)^{r} \hat{t}^{R}, \hat{g}^{r}, \hat{g}^{R}\right)$. After receiving the secret key from PKG, the user can check the key as: $e\left(g, s k_{I D_{0}}\right) \stackrel{?}{=} e\left(g_{1}, \hat{g}_{2}\right) \cdot e\left(h^{I D} f_{1}, s k_{I D_{1}}\right) \cdot e\left(t, s k_{I D_{2}}\right)$.

3) $\operatorname{Enc}\left(I D_{i}, w, m\right)$. Choose $s_{0} \in_{R} \mathbb{Z}_{q}^{*}$ and a one-time signature key pair $\left(K_{s}, K_{v}\right) \leftarrow \operatorname{Sig} . K G\left(1^{k}\right)$, compute $C_{0}=K_{v}, C_{1}=e\left(g_{1}, \hat{g}_{2}\right)^{s_{0}} \cdot m, C_{2}=g^{s_{0}}$, $C_{3}=\left(h^{I D_{i}} f_{1}\right)^{s_{0}}, C_{4}=t^{s_{0}}, C_{5}=\left(h^{w} f_{2}\right)^{s_{0}}$, $C_{6}=\left(h^{K_{v}} f_{3}\right)^{s_{0}}, C_{7}=\operatorname{Sign}\left(K_{s},\left(C_{1}, C_{2}, C_{3}, C_{4}\right.\right.$, $\left.C_{5}, C_{6}\right)$ ), and output the 1-st level ciphertext $C_{1, I D_{i}, w}=$ $\left(C_{0}, C_{1}, C_{2}, C_{3}, C_{4}, C_{5}, C_{6}, C_{7}\right)$, where $I D_{i} \in \mathbb{Z}_{q}^{*}$ and $m \in \mathbb{G}_{T}$.

4) ReKeyGen $\left(I D_{i}, s k_{I D_{i}}, I D_{i^{\prime}}, w\right)$. Choose $\theta_{1}^{(l)} \quad \in_{R}$ $\mathbb{G}_{T}, \quad \rho^{(l)}, s_{1}^{(l)}, \bar{r}_{1}^{(l)} \quad \in_{R} \quad \mathbb{Z}_{q}^{*}$ and a one-time signature key pair $\left(K_{s}^{(l)}, K_{v}^{(l)}\right) \leftarrow \operatorname{Sig} \cdot K G\left(1^{k}\right)$, compute $r k_{w, I D_{i} \rightarrow I D_{i^{\prime}}}: r k_{0}^{(l)}=\left(s k_{I D_{i_{0}}}\left(\hat{h}^{w} \hat{f}_{2}\right)^{\rho^{(l)}}\right)^{H_{1}\left(\theta_{1}^{(l)}\right)}$, $r k_{1}^{(l)}=\left(\hat{g}^{\rho^{(l)}}\right)^{H_{1}\left(\theta_{1}^{(l)}\right)}, r k_{2}^{(l)}=s k_{I D_{i_{1}}}^{H_{1}\left(\theta_{1}^{(l)}\right)}, r k_{3}^{(l)}=$ $s k_{I D_{i_{2}}}^{H_{1}\left(\theta_{(l)}^{(l)}\right)}, r k_{4}^{(l)}=e\left(g_{1}, \hat{g}_{2}\right)^{s_{1}^{(l)}} \cdot \theta_{1}^{(l)}, r k_{5}^{(l)}=g^{s_{1}^{(l)}}$, $r k_{6}^{(l)}=\left(h^{I D_{i^{\prime}}} f_{1}\right)^{s_{1}^{(l)}}, r k_{7}^{(l)}=t^{s_{1}^{(l)}}, r k_{8}^{(l)}=\left(h^{w} f_{2}\right)^{s_{1}^{(l)}}$, $r k_{9}^{(l)}=\left(h^{K_{v}^{(l)}} f_{3}\right)^{s_{1}^{(l)}}, r k_{10}^{(l)}=K_{v}^{(l)}, r k_{11}^{(l)}=\operatorname{Sign}\left(K_{s}^{(l)}\right.$, $\left.\left(r k_{4}^{(l)}, r k_{5}^{(l)}, r k_{6}^{(l)}, r k_{7}^{(l)}, r k_{8}^{(l)}, r k_{9}^{(l)}\right)\right), r k_{12}^{(l)}=$ $\left(h^{I D_{i^{\prime}}} f_{1}\right)^{\bar{r}_{1}^{(l)}}, r k_{13}^{(l)}=g^{\bar{r}_{1}^{(l)}}, r k_{14}^{(l)}=t^{\bar{r}_{1}^{(l)}}, r k_{15}=h^{\bar{r}_{1}^{(l)}}$, $r k_{16}^{(l)}=e\left(g_{1}, \hat{g}_{2}\right)^{\bar{r}_{1}^{(l)}}, r k_{17}^{(l)}=f_{2}^{\bar{r}_{1}^{(l)}}, r k_{18}^{(l)}=f_{3}^{\bar{r}_{1}^{(l)}}$, where $I D_{i}, I D_{i^{\prime}} \in \mathbb{Z}_{q}^{*}$ and $l \in\left\{1, \ldots, \operatorname{poly}\left(1^{k}\right)\right\}$.

5) $\operatorname{Re} \operatorname{Enc}\left(r k_{w, I D_{i} \rightarrow I D_{i^{\prime}}}, w, C_{l, I D_{i}, w}\right)$.

(1) If $l=1$,

i. Verify

$$
\begin{aligned}
& e\left(\hat{h}^{K_{v}} \hat{f}_{3}, C_{2}\right) \stackrel{?}{=} e\left(\hat{g}, C_{6}\right), \\
& e\left(\hat{h}^{w} \hat{f}_{2}, C_{2}\right) \stackrel{?}{=} e\left(\hat{g}, C_{5}\right), \\
& \operatorname{Ver}\left(K_{v}, C_{7},\left(C_{1}, C_{2}, C_{3}, C_{4}, C_{5}, C_{6}\right)\right) \stackrel{?}{=} 1 .
\end{aligned}
$$

If Eq. (1) does not hold, output $\perp$. Otherwise, proceed.

ii. Choose $\theta_{2}^{(1)} \in_{R} \mathbb{G}_{T}, s_{2}^{(1)} \in_{R} \mathbb{Z}_{q}^{*}$ and a one-time signature key pair $\left(\bar{K}_{s}^{(1)}, \bar{K}_{v}^{(1)}\right) \leftarrow \operatorname{Sig} \cdot K G\left(1^{k}\right)$, compute $C_{7}^{(1)}=\frac{e\left(C_{2}, r k_{0}^{(1)}\right) / e\left(C_{5}, r k_{1}^{(1)}\right)}{e\left(C_{3}, r k_{2}^{(1)}\right) \cdot e\left(C_{4}, r k_{3}^{(1)}\right)}, \sigma^{(1)}=$ $S Y M \cdot \operatorname{Enc}\left(C_{0}\left\|C_{1}\right\| \ldots .\left\|C_{7}\right\| C_{7}^{(1)}, H_{2}\left(\theta_{2}^{(1)}\right)\right)$, $C_{8}^{(1)}=r k_{16}^{s_{2}^{(1)}} \cdot \theta_{2}^{(1)}, C_{9}^{(1)}=r k_{13}^{(1) s_{2}^{(1)}}$, $C_{10}^{(1)}=r k_{12}^{(1) s_{2}^{(1)}}, C_{11}^{(1)}=r k_{14}^{(1) s_{2}^{(1)}}, C_{12}^{(1)}=$ $\left(r k_{15}^{(1) w} r k_{17}^{(1)}\right)^{s_{2}^{(1)}}, C_{13}^{(1)}=\left(r k_{15}^{(1) \bar{K}_{v}^{(1)}} r k_{18}^{(1)}\right)^{s_{2}^{(1)}}$, $C_{14}^{(1)}=\bar{K}_{v}^{(1)}, C_{15}^{(1)}=\operatorname{Sign}\left(\bar{K}_{s}^{(1)},\left(C_{8}^{(1)}\right.\right.$, $\left.\left.C_{9}^{(1)}, \quad C_{10}^{(1)}, \quad C_{11}^{(1)}, \quad C_{12}^{(1)}, \quad C_{13}^{(1)}\right)\right) . \quad$ Output $C_{2, I D_{i^{\prime}}, w}=\left(\sigma^{(1)}, C_{8}^{(1)}, C_{9}^{(1)}, C_{10}^{(1)}, C_{11}^{(1)}\right.$, $C_{12}^{(1)}, C_{13}^{(1)}, C_{14}^{(1)}, C_{15}^{(1)}, r k_{4}^{(1)}, r k_{5}^{(1)}, r k_{6}^{(1)}$, $\left.r k_{7}^{(1)}, r k_{8}^{(1)}, r k_{9}^{(1)}, r k_{10}^{(1)}, r k_{11}^{(1)}\right)$.

(2) If $l \geq 2$,

i. Verify

$$
\begin{aligned}
& e\left(r k_{5}^{(l-1)}, \hat{h}^{w} \hat{f}_{2}\right) \stackrel{?}{=} e\left(r k_{8}^{(l-1)}, \hat{g}\right), \\
& e\left(r k_{5}^{(l-1)}, \hat{h}^{K_{v}^{(l-1)}} \hat{f}_{3}\right) \stackrel{?}{=} e\left(r k_{9}^{(l-1)}, \hat{g}\right), \\
& \operatorname{Ver}\left(r k_{10}^{(l-1)}, r k_{11}^{(l-1)},\left(r k_{4}^{(l-1)}, r k_{5}^{(l-1)},\right.\right. \\
& \left.\left.r k_{6}^{(l-1)}, r k_{7}^{(l-1)}, r k_{8}^{(l-1)}, r k_{9}^{(l-1)}\right)\right) \stackrel{?}{=} 1 . \\
& e\left(C_{9}^{(l-1)}, \hat{h}^{w} \hat{f}_{2}\right) \stackrel{?}{=} e\left(C_{12}^{(l-1)}, \hat{g}\right), \\
& e\left(C_{9}^{(l-1)}, \hat{h}^{\bar{K}_{v}(l-1)} \hat{f}_{3}\right) \stackrel{?}{=} e\left(C_{13}^{(l-1)}, \hat{g}\right), \\
& \operatorname{Ver}\left(C_{14}^{(l-1)}, C_{15}^{(l-1)},\left(C_{8}^{(l-1)}, C_{9}^{(l-1)}, C_{10}^{(l-1)},\right.\right. \\
& \left.\left.C_{11}^{(l-1)}, C_{12}^{(l-1)}, C_{13}^{(l-1)}\right)\right) \stackrel{?}{=} 1 .
\end{aligned}
$$

If Eq. (2) and (3) do not hold, output $\perp$. Otherwise, proceed.

ii. Choose $\theta_{2}^{(l)} \quad \in_{R} \quad \mathbb{G}_{T}, \quad s_{2}^{(l)} \quad \in_{R} \quad \mathbb{Z}_{p}^{*}$ and $\left(\bar{K}_{s}^{(l)}, \bar{K}_{v}^{(l)}\right) \leftarrow \operatorname{Sig.KG}\left(1^{k}\right)$, and then compute $C_{7,0}^{(l)}=\frac{e\left(r k_{5}^{(l-1)}, r k_{0}^{(l)}\right) / e\left(r k_{8}^{(l-1)}, r k_{1}^{(l)}\right)}{e\left(r k_{6}^{(l-1)}, r k_{2}^{(l)}\right) \cdot e\left(r k_{7}^{(l-1)}, r k_{3}^{(l)}\right)}$, $C_{7,1}^{(l)}=\frac{e\left(C_{9}^{(l-1)}, r k_{0}^{(l)}\right) / e\left(C_{12}^{(l-1)}, r k_{1}^{(l)}\right)}{e\left(C_{10}^{(l-1)}, r k_{2}^{(l)}\right) \cdot e\left(C_{11}^{(l-1)}, r k_{3}^{(l)}\right)}, \quad \sigma^{(l)}=$ SYM.Enc $\left(\sigma^{(l-1)}\left\|C_{8}^{(l-1)}\right\| \ldots .|| C_{15}^{(l-1)}\left\|r k_{4}^{(l-1)}\right\|\right.$ $\left.\ldots\left\|r k_{11}^{(l-1)}\right\| C_{7,0}^{(l-1)} \| C_{7,1}^{(l-1)}, H_{2}\left(\theta_{2}^{(l)}\right)\right), \quad C_{8}^{(l)}=$ $r k_{16}^{(l) s_{2}^{(l)}} \cdot \theta_{2}^{(l)}, C_{9}^{(l)}=r k_{13}^{(l) s_{2}^{(l)}}, C_{10}^{(l)}=r k_{12}^{(l) s_{2}^{(l)}}$, $C_{11}^{(l)}=r k_{14}^{(l) s_{2}^{(l)}}, C_{12}^{(l)}=\left(r k_{15}^{(l) w} r k_{17}^{(l)}\right)^{s_{2}^{(l)}}$, $C_{13}^{(l)}=\left(r k_{15}^{(l)} \bar{K}_{v}^{(l)} r k_{18}^{(l)}\right)^{s_{2}^{(l)}}, C_{14}^{(l)}=\bar{K}_{v}^{(l)}$, $C_{15}^{(l)}=\operatorname{Sign}\left(\bar{K}_{s}^{(l)},\left(C_{8}^{(l)}, C_{9}^{(l)}, C_{10}^{(l)}, C_{11}^{(l)}, C_{12}^{(l)}\right.\right.$, 
$\left.\left.C_{13}^{(l)}\right)\right)$. Output $C_{l, I D_{i^{\prime}}, w}=\left(\sigma^{(l)}, C_{8}^{(l)}, C_{9}^{(l)}\right.$, $C_{10}^{(l)}, C_{11}^{(l)}, C_{12}^{(l)}, C_{13}^{(l)}, C_{14}^{(l)}, C_{15}^{(l)}, r k_{4}^{(l)}, r k_{5}^{(l)}$, $\left.r k_{6}^{(l)}, r k_{7}^{(l)}, r k_{8}^{(l)}, r k_{9}^{(l)}, r k_{10}^{(l)}, r k_{11}^{(l)}\right)$.

6) $\operatorname{Dec}\left(s k_{I D_{i}}, w, C_{l, I D_{i}, w}\right)$.

(1) If $l=1$,

i. Verify Eq. (1). If Eq. (1) does not hold, output $\perp$. Otherwise, proceed.

ii. Compute

$$
\begin{aligned}
& C_{1} / \frac{e\left(C_{2}, s k_{I D_{0}}\right)}{e\left(C_{3}, s k_{I D_{1}}\right) \cdot e\left(C_{4}, s k_{I D_{2}}\right)} \\
& =e\left(g_{1}, \hat{g}_{2}\right)^{s_{0}} \cdot m / \frac{e\left(g^{s_{0}}, \hat{g}_{0}\left(\hat{h}^{I D_{i}} \hat{f}\right)^{r} \hat{t}^{R}\right)}{e\left(\left(h^{I D_{i}} f\right)^{s_{0}}, \hat{g}^{r}\right) \cdot e\left(t^{s_{0}}, \hat{g}^{R}\right)} \\
& =e\left(g_{1}, \hat{g}_{2}\right)^{s_{0}} \cdot m / e\left(g_{1}, \hat{g}_{2}\right)^{s_{0}}=m .
\end{aligned}
$$

(2) If $l \geq 2$,

i. Verify

$$
\begin{aligned}
& e\left(r k_{5}^{(l)}, \hat{h}^{w} \hat{f}_{2}\right) \stackrel{?}{=} e\left(r k_{8}^{(l)}, \hat{g}\right), \\
& e\left(r k_{5}^{(l)}, \hat{h}^{K_{v}^{(l)}} \hat{f}_{3}\right) \stackrel{?}{=} e\left(r k_{9}^{(l)}, \hat{g}\right), \\
& \operatorname{Ver}\left(r k_{10}^{(l)}, r k_{11}^{(l)},\left(r k_{4}^{(l)}, r k_{5}^{(l)}, r k_{6}^{(l)}, r k_{7}^{(l)}, r k_{8}^{(l)},\right.\right. \\
& \left.\left.r k_{9}^{(l)}\right)\right) \stackrel{?}{=} 1 . \\
& e\left(C_{9}^{(l)}, \hat{h}^{w} \hat{f}_{2}\right) \stackrel{?}{=} e\left(C_{12}^{(l)}, \hat{g}\right), \\
& e\left(C_{9}^{(l)}, \hat{h}^{\bar{K}_{v}^{(l)}} \hat{f}_{3}\right) \stackrel{?}{=} e\left(C_{13}^{(l)}, \hat{g}\right), \\
& \operatorname{Ver}\left(C_{14}^{(l)}, C_{15}^{(l)},\left(C_{8}^{(l)}, C_{9}^{(l)}, C_{10}^{(l)}, C_{11}^{(l)}, C_{12}^{(l)},\right.\right. \\
& \left.\left.C_{13}^{(l)}\right)\right) \stackrel{?}{=} 1 .
\end{aligned}
$$

If Eq. (4) and (5) do not hold, output $\perp$. Otherwise, proceed.

ii. Compute

$$
\begin{aligned}
& \frac{e\left(r k_{5}^{(l)}, s k_{I D_{i_{0}^{\prime}}}\right)}{e\left(r k_{6}^{(l)}, s k_{I D_{i_{1}^{\prime}}}\right) \cdot e\left(r k_{7}^{(l)}, s k_{I D_{i_{2}^{\prime}}}\right)} \\
& =\frac{e\left(g^{s_{1}^{(l)}}, \hat{g}_{0}\left(\hat{h}^{I D_{i^{\prime}}} \hat{f}_{1}\right)^{r} \hat{t}^{R}\right)}{e\left(\left(h^{I D_{i^{\prime}}} f_{1}\right)^{s_{1}^{(l)}}, \hat{g}^{r}\right) \cdot e\left(t^{s_{1}^{(l)}}, \hat{g}^{R}\right)} \\
& =e\left(g_{1}, \hat{g}_{2}\right)^{s_{1}^{(l)}} \text {, }
\end{aligned}
$$

and

$$
\begin{aligned}
& \frac{e\left(C_{9}^{(l)}, s k_{I D_{i_{0}^{\prime}}}\right)}{e\left(C_{10}^{(l)}, s k_{I D_{i_{1}^{\prime}}}\right) \cdot e\left(C_{11}^{(l)}, s k_{I D_{i_{2}^{\prime}}}\right)} \\
& =\frac{e\left(g^{\bar{s}_{2}^{(l)}}, \hat{g}_{0}\left(\hat{h}^{I D_{i^{\prime}}} \hat{f}_{1}\right)^{r} \hat{t}^{R}\right)}{e\left(\left(h^{\left.I D_{i^{\prime}} f_{1}\right)^{\bar{s}_{2}}}, \hat{g}^{r}\right) \cdot e\left(t^{\bar{s}_{2}^{(l)}}, \hat{g}^{R}\right)\right.} \\
& =e\left(g_{1}, \hat{g}_{2}\right)^{\bar{s}_{2}^{(l)}},
\end{aligned}
$$

where $\bar{s}_{2}^{(l)}=s_{2}^{(l)} \cdot \bar{r}_{1}^{(l)}$.

iii. Compute two values $\theta_{1}^{(l)}=r k_{4}^{(l)} / e\left(g_{1}, \hat{g}_{2}\right)^{s_{1}^{(l)}}$, and $\theta_{2}^{(l)}=C_{8}^{(l)} / e\left(g_{1}, \hat{g}_{2}\right)^{\bar{s}_{2}^{(l)}}$. Recover $\sigma^{(l-1)}\left\|C_{8}^{(l-1)}\right\| \ldots .|| C_{15}^{(l-1)}\left\|r k_{4}^{(l-1)}\right\| \ldots\left\|r k_{11}^{(l-1)}\right\|$ $C_{7,0}^{(l-1)} \| C_{7,1}^{(l-1)}=S Y M \cdot \operatorname{Dec}\left(\sigma^{(l)}, H_{2}\left(\theta_{2}^{(l)}\right)\right)$. iv. Compute

$$
\begin{aligned}
& C_{7,0}^{(l-1)\left(H_{1}\left(\theta_{1}^{(l)}\right)\right)^{-1}} \\
& =\left(e\left(g_{1}, \hat{g}_{2}\right)^{s_{1}^{(l-1)}}\right)^{\left(H_{1}\left(\theta_{1}^{(l)}\right)\right)\left(H_{1}\left(\theta_{1}^{(l)}\right)\right)^{-1}} \\
& =e\left(g_{1}, \hat{g}_{2}\right)^{s_{1}^{(l-1)}},
\end{aligned}
$$

and $\theta_{1}^{(l-1)}=r k_{4}^{(l-1)} / e\left(g_{1}, \hat{g}_{2}\right)^{s_{1}^{(l-1)}}$ if E.q (2) holds.

v. Compute

$$
\begin{aligned}
& C_{7,1}^{(l)\left(H_{1}\left(\theta_{1}^{(l)}\right)\right)^{-1}} \\
& =\left(e\left(g_{1}, \hat{g}_{2}\right)^{\bar{s}_{2}^{(l-1)}}\right)^{\left(H_{1}\left(\theta_{1}^{(l)}\right)\right)\left(H_{1}\left(\theta_{1}^{(l)}\right)\right)^{-1}} \\
& =e\left(g_{1}, \hat{g}_{2}\right)^{\bar{s}_{2}^{(l-1)}} \text {, }
\end{aligned}
$$

and $\theta_{2}^{(l-1)}=C_{8}^{(l-1)} / e\left(g_{1}, \hat{g}_{2}\right)^{\bar{s}_{2}^{(l-1)}}$ if E.q (3) holds.

vi. For $1 \leq j \leq l-2$, from $l-2$ to 1 , compute $\theta_{1}^{(j)}$ and $\theta_{2}^{(j)}$ as in the previous steps.

vii. Recover

$$
C_{0}\left\|C_{1}\right\| \ldots . \mid C_{7} \| C_{7}^{(1)}=S Y M . \operatorname{Dec}\left(\sigma^{(1)}, H_{2}\left(\theta_{2}^{(1)}\right)\right) .
$$

\section{Compute}

$$
\begin{aligned}
& C_{1} / C_{7}^{(1)\left(H_{1}\left(\theta_{1}^{(1)}\right)\right)^{-1}} \\
& =e\left(g_{1}, \hat{g}_{2}\right)^{s_{0}} \cdot m / e\left(g_{1}, \hat{g}_{2}\right)^{s_{0} H_{1}\left(\theta_{1}^{(1)}\right)\left(H_{1}\left(\theta_{1}^{(1)}\right)\right)^{-1}} \\
& =m
\end{aligned}
$$

if Eq. (1) holds.

Convert to be single-hop. It is not difficult to convert the current construction to become a single-hop system by eliminating the respective ciphertext and re-encryption key components $C_{12}^{(l)}$ and $r k_{8}^{(l)}$ in the algorithms ReEnc and $R e K e y G e n$, where $l=1$. Without these necessary components, the resulting re-encrypted ciphertext cannot be further converted.

Support multi-condition. The system can be extended to support multi-condition for re-encryption control. We will concatenate all conditions together, and put the resulting concatenation into a TCR hash function $H_{0}$, and further regard the hash value as a keyword exponent $w$.

Theorem 4: Our AMH-IBCPRE scheme is IND-sCon-sIDCCA secure assuming the decisional $\mathcal{P}$-BDH assumption holds, (Sig.KG,Sign, Ver) is a sUF one-time signature scheme, $S Y M$ is a CCA-secure one-time symmetric key encryption and $H_{1}, H_{2}$ are TCR hash functions.

Please refer to Appendix A for the proof of Theorem 4.

Theorem 5: Our AMH-IBCPRE scheme is selective collusion resistant.

Please refer to Appendix B for the proof of Theorem 5.

Theorem 6: Our AMH-IBCPRE scheme achieves ANOOC assuming the decisional $\mathcal{P}$-BDH assumption holds, (Sig.KG,Sign, Ver) is a sUF one-time signature scheme, $S Y M$ is a CCA-secure one-time symmetric key encryption and $H_{1}, H_{2}$ are TCR hash functions.

Please refer to Appendix $\mathrm{C}$ for the proof of Theorem 6. 
Theorem 7: Our AMH-IBCPRE scheme achieves ANORK assuming the decisional $\mathcal{P}$-BDH assumption holds, (Sig.KG,Sign, Ver) is a sUF one-time signature scheme, $S Y M$ is a CCA-secure one-time symmetric key encryption and $H_{1}, H_{2}$ are TCR hash functions.

Please refer to Appendix D for the proof of Theorem 7.

\section{CONCLUSIONS}

We introduced a novel notion, anonymous multi-hop identity-based conditional proxy re-encryption, to preserve the anonymity for ciphertext sender/receiver, conditional data sharing and multiple recipient-update. We further proposed a concrete system for the notion. Meanwhile, we proved the system CCA-secure in the standard model under the decisional $P$-bilinear Diffie-Hellman assumption. To the best of our knowledge, our primitive is the first of its kind in the literature.

\section{REFERENCES}

[1] G. Ateniese, K. Benson, and S. Hohenberger. Key-private proxy reencryption. In CT-RSA, vol. 5473 of $L N C S$, pp. 279-294. Springer, 2009.

[2] G. Ateniese, K. Fu, M. Green, and S. Hohenberger. Improved proxy re-encryption schemes with applications to secure distributed storage. In NDSS, pp. 29-43. Springer, 2005.

[3] G. Ateniese, K. Fu, M. Green, and S. Hohenberger. Improved proxy re-encryption schemes with applications to secure distributed storage. ACM TISSEC, 9(1):1-30, 2006.

[4] M. Bellare and S. Shoup. Two-tier signatures, strongly unforgeable signatures, and fiat-shamir without random oracles. In $P K C$, vol. 4450 of LNCS, pp. 201-216. Springer, 2007.

[5] M. Blaze, G. Bleumer, and M. Strauss. Divertible protocols and atomic proxy cryptography. In EUROCRYPT, pp. 127-144. Springer, 1998.

[6] D. Boneh and X. Boyen. Efficient selective-ID secure identity based encryption without random oracles. In EUROCRYPT, vol. 3027 of LNCS, pp. 223-238. Springer, 2004.

[7] D. Boneh, X. Boyen, and E.-J. Goh. Hierarchical identity based encryption with constant size ciphertext. In EUROCRYPT, vol. 3494 of LNCS, pp. 440-456. Springer, 2005.

[8] X. Boyen and B. Waters. Anonymous hierarchical identity-based encryption (without random oracles). In CRYPTO, vol. 4117 of $L N C S$, pp. 290-307. Springer, 2006.

[9] J. Camenisch, M. Kohlweiss, A. Rial, and C. Sheedy. Blind and anonymous identity-based encryption and authorised private searches on public key encrypted data. In PKC vol. 5443 of $L N C S$, pp. 196-214. Springer, 2009.

[10] R. Canetti, S. Halevi, and J. Katz. Chosen-ciphertext security from identity-based encryption. In Eurocrypt, vol. 3027 of LNCS, pp. $207-$ 222. Springer, 2004.

[11] R. Canetti and S. Hohenberger. Chosen-ciphertext secure proxy reencryption. In CCS, pp. 185-194. ACM, 2007.

[12] C.-K. Chu and W.-G. Tzeng. Identity-based proxy re-encryption without random oracles. In $I S C$, vol. 4779 of $L N C S$, pp. 189-202. Springer, 2007.

[13] R. Cramer and V. Shoup. Design and analysis of practical public-key encryption schemes secure against adaptive chosen ciphertext attack. SIAM J. Comput., 33(1):167-226, January 2004.

[14] L. Ducas. Anonymity from asymmetry: new constructions for anonymous HIBE. In CT-RSA, vol. 5985 of $L N C S$, pp. 148-164. Springer, 2010.

[15] K. Emura, A. Miyaji, and K. Omote. An identity-based proxy reencryption scheme with source hiding property, and its application to a mailing-list system. In EuroPKI, vol. 6711 of LNCS, pp. 77-92. Springer, 2011.

[16] C.-I. Fan, L.-Y. Huang, and P.-H. Ho. Anonymous multireceiver identitybased encryption. Computers, IEEE Transactions on, 59(9):1239-1249, Sept 2010.

[17] M. Green and G. Ateniese. Identity-based proxy re-encryption. In ACNS vol. 4512 of LNCS, pp. 288-306. Springer, 2007.

[18] A. Ivan and Y. Dodis. Proxy cryptography revisited. In NDSS, 2003.
[19] M. Izabachène and D. Pointcheval. New anonymity notions for identitybased encryption. In $S C N$, vol. 5229 of $L N C S$, pp. 375-391. Springer, 2008.

[20] K. Liang, M. H. Au, J. K. Liu, W. Susilo, D. S. Wong, G. Yang, T. V. X Phuong, and Q. Xie. A dfa-based functional proxy re-encryption scheme for secure public cloud data sharing. IEEE Transactions on Information Forensics and Security, 9(10):1667-1680, 2014.

[21] K. Liang, M. H. Au, W. Susilo, D. S. Wong, G. Yang, and Y. Yu. An adaptively CCA-secure ciphertext-policy attribute-based proxy reencryption for cloud data sharing. In ISPEC, vol. 8434 of LNCS, pp. 448-461. Springer, 2014.

[22] K. Liang, C. Chu, X. Tan, D. S. Wong, C. Tang, and J. Zhou. Chosen-ciphertext secure multi-hop identity-based conditional proxy reencryption with constant-size ciphertexts. Theor. Comput. Sci., 539:87$105,2014$.

[23] K. Liang, J. K. Liu, D. S. Wong, and W. Susilo. An efficient cloud-based revocable identity-based proxy re-encryption scheme for public clouds data sharing. In ESORICS, vol. 8712 of LNCS, pp. 257-272. Springer, 2014.

[24] K. Liang, Z. Liu, X. Tan, D. S. Wong, and C. Tang. A CCA-secure identity-based conditional proxy re-encryption without random oracles. In ICISC, vol. 7839 of LNCS, pp. 231-246. Springer, 2012.

[25] B. Libert and D. Vergnaud. Unidirectional chosen-ciphertext secure proxy re-encryption. In $P K C$, vol. 4939 of $L N C S$, pp. 360-379. Springer, 2008.

[26] R. Lu, X. Lin, J. Shao, and K. Liang. RCCA-secure multi-use bidirectional proxy re-encryption with master secret security. In ProvSec, vol. 8782 of $L N C S$, pp. 194-205. Springer, 2014.

[27] M. Mambo and E. Okamoto. Proxy cryptosystems: Delegation of the power to decrypt ciphertexts. IEICE Transactions, E80-A(1):54-63, 1997.

[28] T. Matsuo. Proxy re-encryption systems for identity-based encryption. In Pairing, vol. 4575 of LNCS, pp. 247-267. Springer, 2007.

[29] T. Mizuno and H. Doi. Secure and efficient IBE-PKE proxy reencryption. IEICE Transactions, E94-A(1):36-44, 2011.

[30] Y. Rao and R. Dutta. Recipient anonymous ciphertext-policy attribute based encryption. In Information Systems Security, vol. 8303 of LNCS, pp. 329-344. Springer, 2013.

[31] J. Shao. Anonymous id-based proxy re-encryption. In ACISP, vol. 7372 of LNCS, pp. 364-375. Springer, 2012.

[32] J. Shao and Z. Cao. Multi-use unidirectional identity-based proxy reencryption from hierarchical identity- based encryption. Inform. Sci., 2012.

[33] J. Shao, P. Liu, G. Wei, and Y. Ling. Anonymous proxy reencryption. Security and Communication Networks, 5(5):439-449, May 2012.

[34] J. Shao, P. Liu, and Y. Zhou. Achieving key privacy without losing CCA security in proxy re-encryption. The Journal of Systems and Software, 2011. http://doi:10.1016/j.jss.2011.09.034.

[35] Q. Tang, P. Hartel, and W. Jonker. Information security and cryptology. chapter Inter-domain Identity-Based Proxy Re-encryption, pages 332347. Springer, 2009.

[36] H. Wang, Z. Cao, and L. Wang. Multi-use and unidirectional identity-based proxy re-encryption schemes. Information Sciences, 180(20):4042-4059, 2010.

[37] L. Wang, L. Wang, M. Mambo, and E. Okamoto. Identity-based proxy cryptosystems with revocability and hierarchical confidentialities. IEICE Transactions, 95-A(1):70-88, 2012.

[38] B. Waters. Efficient identity-based encryption without random oracles. In EUROCRYPT, vol. 3494 of $L N C S$, pp. 114-127. Springer, 2005.

[39] B. Waters. Dual system encryption: Realizing fully secure IBE and HIBE under simple assumptions. In CRYPTO, vol. 5677 of LNCS, pp. 619-636. Springer, 2009.

[40] Y. Zhang, X. Chen, J. Li, D. S. Wong, and H. Li. Anonymous attributebased encryption supporting efficient decryption test. In $C C S$, pp. 511516. ACM, 2013.

\section{APPENDIX}

\section{A. Proof of Theorem 4}

Proof: Suppose there is an adversary $\mathcal{A}$ who can break the IND-sCon-sID-CCA security of our scheme. We then construct a reduction algorithm $\mathcal{B}$ to break the CCA security of 3-level Du-ANO-HIBE. Let $\mathcal{B}_{1}$ be the challenger of the 
3-level Du-ANO-HIBE in the CCA experiment. $\mathcal{B}$ maintains the following tables which are initially empty.

1) $D C T$ : records the tuples $\left(w \mid I D_{i}, \ldots, I D_{j}, t a g\right)$, which are the delegation chains under condition $w$ from $I D_{i}$ to $I D_{j}$, where $\operatorname{tag}$ denotes that the chain is either uncorrupted ("1") or corrupted ("0"), $i, j \in\left\{1, \ldots, \operatorname{poly}\left(1^{k}\right)\right\}$.

2) $S K T$ : records the tuples $\left(I D_{i}, s k_{I D_{i}}\right)$, which are the information of the secret keys (obtained in the simulation).

3) $R K T$ : records the tuples $\left(I D_{i}, I D_{i^{\prime}}, w, r k_{w, I D_{i} \rightarrow I D_{i^{\prime}}}\right.$, $\left.\theta_{1}\right)$, which are the results of the queries to $\mathcal{O}_{r k}\left(I D_{i}, I D_{i^{\prime}}, w\right)$, where tag denotes that the reencryption key is either a valid key (" 1 ") or a random key ("0").

4) $R E T$ : records the tuples $\left(I D_{i}, I D_{i^{\prime}}, w, C_{\left(l+1, I D_{i^{\prime}}, w\right)}\right.$, $\operatorname{tag})$, which are the results of the queries to $\mathcal{O}_{r e}\left(I D_{i}\right.$, $\left.I D_{i^{\prime}}, w, C_{\left(l, I D_{i}, w\right)}\right)$, where tag denotes that the reencrypted ciphertext is generated under a valid reencryption key (" 1 "), a random key (" 0 ") or generated without using any re-encryption key (" $\perp$ ”).

1) Init. $\mathcal{A}$ outputs $I D^{*}$ and $w^{*}$ to $\mathcal{B}, \mathcal{B}$ then forwards them as well as a self-chosen $K_{v}^{* 2}$ to $\mathcal{B}_{1}$.

2) Setup. $\mathcal{B}_{1}$ sends $m p k=\left(g, \hat{g}, g_{1}, h, f_{1}, f_{2}, f_{3}, t, \hat{g}_{2}, \hat{f}_{2}\right.$, $\hat{f}_{3}, \hat{h},($ Sig.KG, Sign, Ver $\left.)\right)$ to $\mathcal{B}$. Then $\mathcal{B}$ chooses two TCR hash function $H_{1}, H_{2}$ and a CCA-secure one-time symmetric key encryption $S Y M$ as in the real scheme, adds them to $m p k$ and forwards the resulting $m p k$ to $\mathcal{A}$.

3) Phase 1. $\mathcal{A}$ issues a series of queries.

a) $\mathcal{O}_{s k}(I D)$ : if there is a tuple $\left(I D, s k_{I D}\right)$ in $S K T, \mathcal{B}$ returns $s k_{I D}$ to $\mathcal{A}$. Otherwise, $\mathcal{B}$ works as follows.

- If $I D^{*}=I D$ or $I D$ is in $\left(w^{*} \mid I D^{*}, \ldots, 1\right) \in D C T$ holds, $\mathcal{B}$ outputs $\perp$.

- Otherwise, $\mathcal{B}$ forwards the query to the secret key extraction oracle of 3-level Du-ANO-HIBE, $\mathcal{O}_{\text {extract }}$, obtains the secret key and forwards the key to $\mathcal{A}$. Finally, $\mathcal{B}$ adds $\left(I D, s k_{I D}\right)$ to $S K T$.

b) $\mathcal{O}_{r k}\left(I D_{i}, I D_{i^{\prime}}, w\right)$ : if there is a tuple $\left(I D_{i}\right.$, $\left.I D_{i^{\prime}}, w, r k_{w, I D_{i} \rightarrow I D_{i^{\prime}}}, \theta_{1}^{(l)}, *\right)$ in $R K T, \mathcal{B}$ returns $r k_{w, I D_{i} \rightarrow I D_{i^{\prime}}}$ to $\mathcal{A}$. Otherwise, $\mathcal{B}$ works as follows.

- If $\left(I D^{*}=I D_{i}\right.$ or $I D_{i}$ in $\left(w^{*} \mid I D^{*}, \ldots, 1\right) \in$ $D C T) \wedge I D_{i^{\prime}}$ in $\left(w^{*} \mid *, \ldots, 0\right) \in D C T$ hold, then $\mathcal{B}$ outputs $\perp$, where $w^{*}=w$.

- If $I D^{*}=I D_{i} \wedge I D_{i^{\prime}}$ in $\left(w^{*} \mid *, \ldots, 1\right) \in D C T$ hold, $\mathcal{B}$ sets $r k_{0}^{(l)}=\sigma_{1}, r k_{1}^{(l)}=\sigma_{2}, r k_{2}^{(l)}=\sigma_{3}, r k_{3}^{(l)}=$ $\sigma_{4}$, and constructs the rest of components as in the real scheme, where $\sigma_{1}, \sigma_{2}, \sigma_{3}, \sigma_{4} \in_{R} \mathbb{G}_{2}, w^{*}=w$. $\mathcal{B}$ sends the re-encryption key to $\mathcal{A}$, and adds $\left(I D_{i}\right.$, $\left.I D_{j}, w, r k_{w, I D_{i} \rightarrow I D_{i^{\prime}}}, \theta_{1}^{(l)}, 0\right)$ to $R K T$.

- If $I D^{*}=I D_{i} \wedge w^{*} \neq w$ hold, $\mathcal{B}$ sends $I D=\left(I D^{*}, w\right)$ to $\mathcal{O}_{\text {extract }}$, and obtains $s k_{I D}$ which are identical to $r k_{0}^{(l) H_{1}\left(\theta_{1}^{(l)}\right)^{-1}}, r k_{1}^{(l) H_{1}\left(\theta_{1}^{(l)}\right)^{-1}}$, $r k_{2}^{(l) H_{1}\left(\theta_{1}^{(l)}\right)^{-1}}$ and $r k_{3}^{(l) H_{1}\left(\theta_{1}^{(l)}\right)^{-1}} \cdot \mathcal{B}$ then generates the rest of components of the re-encryption key as in the real scheme, and adds $\left(I D_{i}, I D_{j}, w\right.$, $\left.r k_{w, I D_{i} \rightarrow I D_{i^{\prime}}}, \theta_{1}^{(l)}, 1\right)$ to $R K T$.

${ }^{2}$ Note this verification key will not be used in the query phases but in the challenge phase.
- Otherwise, $\mathcal{B}$ queries $I D_{i}$ to $\mathcal{O}_{\text {extract }}$ to obtain the secret key $s k_{I D_{i}}$, next generates the reencryption key as in the real scheme and responds the key to $\mathcal{A}$, and finally adds $\left(I D_{i}, s k_{I D_{i}}\right)$ and $\left(I D_{i}, I D_{i^{\prime}}, w, r k_{w, I D_{i} \rightarrow I D_{i^{\prime}}}, \theta_{1}^{(l)}, 1\right)$ to $S K T$ and $R K T$, respectively, where $\theta_{1}^{(l)} \in_{R} \mathbb{G}_{T}$. Note if $\left(I D_{i}, s k_{I D_{i}}\right)$ is in $S K T, \mathcal{B}$ uses $s k_{I D_{i}}$ to generate the re-encryption key as in the real scheme.

c) $\mathcal{O}_{r e}\left(I D_{i}, I D_{i^{\prime}}, w, C_{l, I D_{i}, w}\right)$ :

- If the first case of step b) does not hold, $\mathcal{B}$ can first construct the re-encryption key as in step b) and then generate the re-encrypted ciphertext using the re-encryption key. Finally, $\mathcal{B}$ responds the ciphertext to $\mathcal{A}$ and adds $\left(I D_{i}, I D_{j}, w, r k_{w, I D_{i} \rightarrow I D_{i^{\prime}}}, \theta_{1}^{(l)}, *\right)$ and $\left(I D_{i}, I D_{i^{\prime}}, w, C_{\left(l+1, I D_{i^{\prime}}, w\right)}, *\right)$ to $R K T$ and $R E T$, respectively.

- Otherwise

i) If $l=1, \mathcal{B}$ first verifies whether Eq. (1) holds. If not $\mathcal{B}$ outputs $\perp$. Otherwise, $\mathcal{B}$ queries $\left.\left(\left(I D_{i}, w, K_{v}\right), C_{1, I D_{i}, w}\right)\right)$ to the decryption oracle of 3-level Du-ANO-HIBE, denoted as $\mathcal{O}_{\text {decrypt }}$, and obtains the underlying message. With knowledge of the message, $\mathcal{B}$ can recover the hiding factor $K_{0}=e\left(g_{1}, \hat{g}_{2}\right)^{s_{0}}$. $\mathcal{B}$ further calculates $C_{7}^{(1)}=K_{0}^{H_{1}\left(\theta_{1}^{(1)}\right)}$, constructs symmetric encryption $\sigma^{(1)}$ with $\theta_{2}^{(1)}$, generates the ciphertext $C_{8}^{(1)}, \ldots, C_{15}^{(1)}$ under $I D_{i^{\prime}}$ to hide $\theta_{2}^{(1)}$ and the ciphertext $r k_{4}^{(1)}, \ldots, r k_{11}^{(1)}$ under $I D_{i^{\prime}}$ to hide $\theta_{1}^{(1)}$ as in the real scheme, where $\theta_{1}^{(1)}, \theta_{2}^{(1)} \in_{R} \mathbb{G}_{T}$. Finally, $\mathcal{B}$ responds the reencrypted ciphertext to $\mathcal{A}$ and adds $\left(I D_{i}, I D_{i^{\prime}}\right.$, $\left.w, C_{\left(2, I D_{i^{\prime}}, w\right)}, \perp\right)$ to $R E T$.

ii) If $l \geq 2, \mathcal{B}$ first verifies whether Eq. (2) and Eq. (3) hold. If not $\mathcal{B}$ outputs $\perp$. Otherwise, $\mathcal{B}$ constructs the corresponding re-encrypted ciphertext in the identical method as above except that $C_{7,0}^{(l)}, C_{7,1}^{(l)}$ should be generated like the way of generating $C_{7}^{(1)}$.

Note the queries issued by $\mathcal{A}$ should follow the restrictions defined in Definition 2.

d) $\mathcal{O}_{\text {dec }}\left(I D_{i}, w, C_{l, I D_{i}, w}\right)$ : if $C_{l, I D_{i}, w}$ is a derivative of the challenge ciphertext, $\mathcal{B}$ outputs $\perp$. Since $\mathcal{B}$ can access to the decryption oracle $\mathcal{O}_{\text {decrypt }}$, then it can easily tell any derivative.

- If $l=1$, that is, $C_{1, I D_{i}, w}$ is the first level ciphertext without any re-encryption. $\mathcal{B}$ first verifies whether Eq. (1) holds. If not, $\mathcal{B}$ outputs $\perp$ and proceeds otherwise.

i) If $\left(I D_{i}, s k_{I D_{i}}\right) \in S K T$, then $\mathcal{B}$ recovers $m$ using $s k_{I D_{i}}$ as in the real scheme.

ii) Otherwise, $\mathcal{B}$ queries $\left.\left(\left(I D_{i}, w, K_{v}\right), C_{1, I D_{i}, w}\right)\right)$ to $\mathcal{O}_{\text {decrypt }}$, and returns $m$.

- If $l \geq 2$, that is, $C_{l, I D_{i}, w}$ is the re-encrypted ciphertext. $\mathcal{B}$ first verifies whether Eq. (4) and Eq. (5) hold. If not, $\mathcal{B}$ outputs $\perp$ and proceeds otherwise. 
i) If $w^{*}=w$ and $I D^{*}=I D_{i}, \mathcal{B}$ issues $I D=$ $\left(I D^{*}, w^{*}, \bar{K}_{v}^{(l)}\right)$ to $\mathcal{O}_{\text {extract }}$, and obtains $s k_{I D}$. $\mathcal{B}$ then recovers $\theta_{1}^{(l)}, \theta_{2}^{(l)}$ as in the algorithm $D e c$ of 3-level Du-ANO-HIBE, and further recovers $m$ as in the real scheme.

ii) Else, $\mathcal{B}$ forwards $\left(r k_{4}^{(l)}, \ldots, r k_{11}^{(l)}\right)$ and $\left(C_{8}^{(l)}, \ldots, C_{15}^{(l)}\right)$ to $\mathcal{O}_{\text {decrypt }}$ and then obtains $\theta_{1}^{(l)}, \theta_{2}^{(l)} . \mathcal{B}$ uses $\theta_{1}^{(l)}$ and $\theta_{2}^{(l)}$ to recover $\theta_{1}^{(i)}, \theta_{2}^{(i)}$ for $1 \leq i \leq l-1$ from $l-1$ to 1 . Next, $\mathcal{B}$ recovers $\left(C_{0}, \ldots, C_{7}\right)$ by using $\theta_{2}^{(1)}$, computes $K_{0}$ with $\theta_{1}^{(1)}$, and finally recovers $m$ as in the real scheme.

Note $\mathcal{B}$ can recover $\theta_{1}^{(i)}, \theta_{2}^{(i)}$ on its own if $\left(I D_{i}, s k_{I D_{i}}\right) \in S K T$ for any $i, 1 \leq i \leq l$.

4) Challenge. When $\mathcal{A}$ decides that Phase 1 is over, it outputs $m_{0}, m_{1}$ and $\left\{I D_{i_{j}}\right\}_{1}^{j=l^{*}-1}$ to $\mathcal{B}$. $\mathcal{B}$ first generates the ciphertext $C_{l^{*}-1, I D_{i_{l^{*}-1}}, w^{*}}$ for $m_{b}$ as in the real scheme, where all re-encryption keys and the first level ciphertext $C_{1, I D_{i_{1}}, w^{*}}$ can be easily constructed with knowledge of $s k_{I D_{i_{j}}}$ (which can be obtained from $\mathcal{O}_{\text {extract }}$ ), and $b \in\{0,1\}$. $\mathcal{B}$ further chooses $\left(\theta_{1,0}^{\left(l^{*}\right)}, \theta_{1,1}^{\left(l^{*}\right)}\right),\left(\theta_{2,0}^{\left(l^{*}\right)}, \theta_{2,1}^{\left(l^{*}\right)}\right) \in_{R} \mathbb{G}_{T}$, and forwards them to $\mathcal{B}_{1} . \mathcal{B}_{1}$ returns $r k_{4}^{\left(l^{*}\right)}, \ldots ., r k_{11}^{\left(l^{*}\right)}$ and $C_{8}^{\left(l^{*}\right)}, \ldots ., C_{15}^{\left(l^{*}\right)}$ for $\theta_{1, \hat{b}}^{\left(l^{*}\right)}$ and $\theta_{2, \bar{b}}^{\left(l^{*}\right)}$, respectively, where $\hat{b}, \bar{b} \in\{0,1\} . \mathcal{B}$ then generates the re-encryption key $r k_{w^{*}, I D_{i_{l^{*}}-1} \rightarrow I D^{*}}$ components $r k_{0}^{\left(l^{*}\right)}, r k_{1}^{\left(l^{*}\right)}, r k_{2}^{\left(l^{*}\right)}, r k_{3}^{\left(l^{*}\right)}$ (with $\theta_{1, \hat{b}}^{\left(l^{*}\right)}$ ), and $C_{7,0}^{\left(l^{*}\right)}, C_{7,1}^{\left(l^{*}\right)}, \sigma^{\left(l^{*}\right)}$ (with $\theta_{2, \hat{b}}^{\left(l^{*}\right)}$ ) as in the real scheme. $\mathcal{B}$ finally returns $C_{l^{*}, I D^{*}, w^{*}}$ to $\mathcal{A}$.

5) Phase 2. Same as in Phase 1.

6) Guess. $\mathcal{B}$ outputs whatever $\mathcal{A}$ outputs.

In the above simulations, $\mathcal{A}$ 's view is identical to the real attack except for the case that $\mathcal{B}$ responds the random re-encryption keys in step (b) to $\mathcal{A}$. It is clear that the components (of the random re-encryption key) $\sigma_{1}, \sigma_{2}, \sigma_{3}, \sigma_{4}$ can take the form of $r k_{0}^{(l)}, r k_{1}^{(l)}, r k_{2}^{(l)}, r k_{3}^{(l)}$ (of the real reencryption key), respectively. Besides, $r k_{12}^{(l)}, \ldots, r k_{18}^{(l)}$ of the random re-encryption key are identical to those of the real one. Hence, the problem is equivalent to the distinguishability of the encryption of a random $\theta_{1}^{(l)}$ (of the random re-encryption key) and the encryption of the $\theta_{1}^{(l)}$ (of the real re-encryption key). If there is an adversary $\mathcal{A}_{1}$ who can distinguish the encryptions above, we can construct an algorithm $\mathcal{B}_{2}$ to break the decisional $\mathcal{P}$-BDH problem in $\left(\mathbb{G}_{1}, \mathbb{G}_{2}\right)$ by using $\mathcal{A}_{1}$.

$\mathcal{B}$ chooses a challenge verification key $K_{v}^{*}$ beforehand, and this verification key cannot be used in the simulations. Therefore, $\mathcal{B}$ 's advantage is at least $\frac{\epsilon\left(q_{r k}+q_{r e}+q_{d e c}\right)}{4 q}$, and the running time of $\mathcal{B}$ is $O($ time $(\mathcal{A}))$, where $q_{r k}, q_{r e}, q_{d e c}$ are the total numbers of re-encryption key extraction, re-encryption and decryption queries, respectively.

\section{B. Proof of Theorem 5}

Below we prove that the IND-sCon-sID-CCA security of our scheme already implies the selective collusion resistance.

Proof: In the game of Definition 2, an adversary $\mathcal{A}$ is allowed to gain access to the re-encryption keys $r k_{w, I D^{*} \rightarrow I D_{i^{\prime}}}$ and $r k_{w, I D_{i^{\prime}} \rightarrow I D_{i^{\prime \prime}}}$, where $w$ is not the challenge condition, $I D_{i^{\prime}}$ is honest and $I D_{i^{\prime \prime}}$ is corrupted by $\mathcal{A}$. Suppose our AMH-IBCPRE system is not collusion resistant, $\mathcal{A}$ can compromise the secret key $s k_{I D_{i^{\prime}}}$ with knowledge of $s k_{I D_{i^{\prime \prime}}}$ and $r k_{w, I D_{i^{\prime}} \rightarrow I D_{i^{\prime \prime}}} . \mathcal{A}$ further compromise $s k_{I D^{*}}$ with knowledge of $s k_{I D_{i^{\prime}}}$ and $r k_{w, I D^{*} \rightarrow I D_{i^{\prime}}}$. Given the challenge ciphertext $C_{l^{*}, I D^{*}, w^{*}}$, the adversary $\mathcal{A}$ can easily retrieve the value of the bit $b$ by using $s k_{I D^{*}}$. The IND-sCon-sID-CCA security fails here that contradicts our security notion. Therefore, the IND-sCon-sID-CCA security implies collusion resistance.

\section{Proof of Theorem 6}

Proof: Suppose there is an adversary $\mathcal{A}$ who can break the ANO-OC security of our scheme, then we can construct an algorithm $\mathcal{B}$ to solve the decisional $\mathcal{P}$-BDH problem in $\left(\mathbb{G}_{1}, \mathbb{G}_{2}\right)$ by using $\mathcal{A}$.

- Init. Same as the proof of Theorem 4 except the followings. $\mathcal{A}$ outputs $I D_{0}^{*}$ and $I D_{1}^{*}$ to $\mathcal{B}$, and $\mathcal{B}$ forwards $I D_{b}^{*}$ to $\mathcal{B}_{1}$, where $b \in\{0,1\}$.

- Setup. Same as the proof of Theorem 4.

- Phase 1. Same as the proof of Theorem 4.

- Challenge. When $\mathcal{A}$ decides that Phase 2 is over, then it outputs $m$ to $\mathcal{B}$. $\mathcal{B}$ chooses a random message $m^{\prime}$ fro message space, and sets $m_{1}=m, m_{0}=m^{\prime} . \mathcal{B}$ next forwards $m_{0}, m_{1}$ to $\mathcal{B}_{1}$, obtains the ciphertext $C_{1, I D_{b}^{*}, w^{*}}$ for $m_{\bar{b}}$ from $\mathcal{B}_{1}$, where $\bar{b} \in\{0,1\}$. Then $\mathcal{B}$ forwards $C_{1, I D_{b}^{*}, w^{*}}$ to $\mathcal{A}$.

- Phase 2. Same as Phase 1.

- Guess. $\mathcal{B}$ outputs whatever $\mathcal{A}$ outputs.

The probability analysis is the same as that of Theorem 4. Therefore, the advantage of $\mathcal{B}$ is at least $\frac{\operatorname{Adv}_{\mathcal{A}}^{A N O-O C}\left(1^{k}\right)\left(q_{r k}+q_{r e}+q_{d e c}\right)}{2 q}$, and the running time of $\mathcal{B}$ is $O(\operatorname{time}(\mathcal{A}))$.

\section{Proof of Theorem 7}

Proof: Supposing there is an adversary $\mathcal{A}$ who can break the ANO-RK security of our scheme, we can construct an algorithm $\mathcal{B}$ to solve the decisional $\mathcal{P}$-BDH problem in $\left(\mathbb{G}_{1}, \mathbb{G}_{2}\right)$ by using $\mathcal{A}$.

- Init. Same as the proof of Theorem 4 except that $\mathcal{A}$ outputs $I D^{\prime}, I D^{*}$ to $\mathcal{B}$, and $\mathcal{B}$ next forwards $I D^{*}$ to $\mathcal{B}_{1}$.

- Setup. Same as the proof of Theorem 4.

- Phase 1. $\mathcal{A}$ is allowed to issue queries to the oracles $\mathcal{O}_{s k}, \mathcal{O}_{r k}, \mathcal{O}_{r e}, \mathcal{O}_{d e c}$ as in the Phase 1 of the proof of Theorem 4.

- Challenge. When $\mathcal{A}$ decides that Phase 1 is over, $\mathcal{B}$ flips a coin $b \in\{0,1\}$. If $b=0, \mathcal{B}$ sets $r k_{0}^{(l)}=$ $\sigma_{1}^{H_{1}\left(\theta_{1, \hat{b}}^{(l)}\right)}, r k_{1}^{(l)}=\sigma_{2}^{H_{1}\left(\theta_{1, \hat{b}}^{(l)}\right)}, r k_{2}^{(l)}=\sigma_{3}^{H_{1}\left(\theta_{1, \hat{b}}^{(l)}\right)}, r k_{3}^{(l)}=$ $\sigma_{4}^{H_{1}\left(\theta_{1, \hat{b}}^{(l)}\right)}$, and issues $\theta_{1,0}^{(l)}, \theta_{1,1}^{(l)} \in R \quad \mathbb{G}_{T}$ to $\mathcal{B}_{1}$, where $\sigma_{1}, \sigma_{2}, \sigma_{3}, \sigma_{4} \in \mathbb{G}_{2}$ and $\hat{b} \in\{0,1\} . \mathcal{B}_{1}$ returns $r k_{4}^{(l)}, \ldots$, $r k_{11}^{(l)}$ for $\theta_{1, \hat{b}}^{(l)}$. $\mathcal{B}$ next constructs the rest of re-encryption key's components (i.e. $\left.r k_{12}^{(l)}, \ldots, r k_{18}^{(l)}\right)$ as in the real scheme. That is, such a re-encryption key is a random key from the re-encryption key space. Otherwise, $\mathcal{B}$ constructs 
the re-encryption key $r k_{w^{*}, I D^{\prime} \rightarrow I D^{*}}$ as above except that $r k_{0}^{(l)}, r k_{1}^{(l)}, r k_{2}^{(l)}, r k_{3}^{(l)}$ are constructed as in the real scheme with knowledge $s k_{I D^{\prime}}$ which can be obtained from $\mathcal{O}_{\text {extract }}$. Finally, $\mathcal{B}$ responds $r k_{w^{*}, I D^{\prime} \rightarrow I D^{*}}$ to $\mathcal{A}$.

- Phase 2. Same as Phase 1.

- Guess. $\mathcal{B}$ outputs whatever $\mathcal{A}$ outputs.

Similar to the analysis in the proof of Theorem $6, \mathcal{B}$ 's advantage is at least $\frac{A d v_{\mathcal{A}}^{A N O-R K}\left(1^{k}\right)\left(q_{r k}+q_{r e}+q_{d e c}\right)}{2 q}$, and the running time of $\mathcal{B}$ is $O(\operatorname{time}(\mathcal{A}))$. 